\title{
The ultra-hot-Jupiter KELT-16 b: Dynamical Evolution and Atmospheric Properties
}

\author{
L. Mancini ${ }^{1,2,3,4 \star}$, J. Southworth ${ }^{5}$, L. Naponiello ${ }^{1,6}$, Ö. Baştürk ${ }^{7}$, D. Barbato ${ }^{8,3}$, F. Biagiotti ${ }^{1}$, I. Bruni ${ }^{9}$, \\ L. Cabona ${ }^{10}$, G. D’Ago ${ }^{11}$, M. Damasso ${ }^{3}$, A. Erdem ${ }^{12,13}$, D. Evans ${ }^{5}$, Th. Henning ${ }^{2}$, O. Öztürk ${ }^{12,13}$, D. Ricci ${ }^{14}$,
} A. Sozzetti ${ }^{3}$, J. Tregloan-Reed ${ }^{15}$, S. Yalçınkaya ${ }^{7}$

${ }^{1}$ Department of Physics, University of Rome "Tor Vergata”, Via della Ricerca Scientifica 1, I-00133, Rome, Italy

${ }^{2}$ Max Planck Institute for Astronomy, Königstuhl 17, D-69117, Heidelberg, Germany

${ }^{3}$ INAF - Turin Astrophysical Observatory, via Osservatorio 20, I-10025, Pino Torinese, Italy

${ }^{4}$ International Institute for Advanced Scientific Studies (IIASS), Via G. Pellegrino 19, I-84019, Vietri sul Mare (SA), Italy

${ }^{5}$ Astrophysics Group, Keele University, Keele ST5 5BG, UK

${ }^{6}$ Department of Physics and Astronomy, University of Florence, Via Sansone 1, I-50019, Sesto Fiorentino (FI), Italy

${ }^{7}$ Ankara University, Faculty of Science, Astronomy \& Space Sciences Department, Tandogan, TR-06100, Ankara, Turkey

${ }^{8}$ Observatoire de Genève, UniversitÃl' de Genève, 51 Chemin Pegasi, 1290 Sauverny, Switzerland

${ }^{9}$ INAF - OAS, Osservatorio di Astrofisica e Scienza dello Spazio di Bologna, Via P. Gobetti 93/3, I-40129, Bologna, Italy

${ }^{10}$ INAF - Brera Astronomical Observatory, Via E. Bianchi 46, I-23807, Merate (LC), Italy

${ }^{11}$ Instituto de Astrofísica, Pontificia Universidad Católica de Chile, Av. Vicuña Mackenna 4860, 7820436 Macul, Santiago, Chile

${ }^{12}$ Department of Physics, Faculty of Arts and Sciences, Çanakkale Onsekiz Mart University, Terzioğlu Kampüsü, TR-17020, Çanakkale, Turkey

${ }^{13}$ Astrophysics Research Centre and Observatory, Çanakkale Onsekiz, Mart University, Terzioğlu KampÃ ijs $\tilde{A} i j$, , TR-17020, Çanakkale, Turkey

${ }^{14}$ INAF - Padova Astronomical Observatory, Vicolo dell'Osservatorio 5, I-35122, Padova, Italy

${ }^{15}$ Instituto de Investigación en Astronomia y Ciencias Planetarias, Universidad de Atacama, Copiapó, Atacama, Chile

Accepted XXX. Received YYY; in original form ZZZ

\begin{abstract}
We present broad-band photometry of 30 planetary transits of the ultra-hot Jupiter KELT-16 b, using five medium-class telescopes. The transits were monitored through standard $B, V, R, I$ filters and four were simultaneously observed from different places, for a total of 36 new light curves. We used these new photometric data and those from the TESS space telescope to review the main physical properties of the KELT-16 planetary system. Our results agree with previous measurements but are more precise. We estimated the mid-transit times for each of these transits and combined them with others from the literature to obtain 69 epochs, with a time baseline extending over more than four years, and searched for transit time variations. We found no evidence for a period change, suggesting a lower limit for orbital decay at $8 \mathrm{Myr}$, with a lower limit on the reduced tidal quality factor of $Q_{\star}^{\prime}>(1.9 \pm 0.8) \times 10^{5}$ with $95 \%$ confidence. We built up an observational, low-resolution transmission spectrum of the planet, finding evidence of the presence of optical absorbers, although with a low significance. Using TESS data, we reconstructed the phase curve finding that KELT-16 b has a phase offset of $25.25 \pm 14.03{ }^{\circ} \mathrm{E}$, a day- and night-side brightness temperature of $3190 \pm 61 \mathrm{~K}$ and $2668 \pm 56 \mathrm{~K}$, respectively. Finally, we compared the flux ratio of the planet over its star at the TESS and Spitzer wavelengths with theoretical emission spectra, finding evidence of a temperature inversion in the planet's atmosphere, the chemical composition of which is preferably oxygen-rich rather than carbon-rich.
\end{abstract}

Key words: planetary systems - stars: fundamental parameters - stars: individual: KELT-16 - techniques: photometric - method: data analysis

\section{INTRODUCTION}

Although hot Jupiters were the first exoplanets that have been discovered more than twenty years ago (Mayor \& Queloz 1995), there are still many open questions related to this class of planets. It is not well established, for example, the predominant physical mechanism that caused these gas planets to form, as well as how did they come

^ E-mail:Imancini@roma2.infn.it to migrate and occupy such short-period orbits ( $\sim 1$ day) around their host stars ${ }^{1}$. The future evolution of a hot Jupiter is also unclear and difficult to predict exactly but is surely linked to that of its star and its interaction with it. This because stellar tidal forces become

\footnotetext{
1 The two leading theories, which can explain the shrinking of the orbit of a giant planet, are $(i)$ early interactions between a giant planet and the protoplanetary disk of its parent star and (ii) planet-planet scattering.
} 
particularly relevant when a giant planet is at a distance of roughly 0.1 au from its host star.

The gravitational interaction forces a hot Jupiter to change its rotation rate and circularise its orbit as a result of energy exchange and heat dissipation. This can lead to a spin-orbit synchronization and eventually to orbital decay (e.g., Hut 1980; Rasio \& Ford 1996; Sasselov 2003; Ferraz-Mello et al. 2008; Levrard et al. 2009). When the planet approaches the Roche limit, it begins to lose mass, in favour of its star, via a mechanism known as Roche-lobe overflow. At the Roche limit, tidal forces overcome the gravity of the planet itself, which may then be partially or totally disrupted (Dosopoulou et al. 2017). In case of planetary systems, the Roche-limit separation can be written as $a_{\mathrm{R}} \approx 2.165 R_{\mathrm{p}}\left(M_{\star} / M_{\mathrm{p}}\right)^{1 / 3}$ (Paczynski 1971; Matsumura et al. 2010).

The frictional processes in the star lead to the dissipation of orbital energy, whose efficiency can be parameterised by the tidal dissipation quality factor, $Q_{\star}$, which can be expressed as the ratio between the energy in the orbit of a binary system and the amount of energy lost in each orbit due to internal friction (Goldreich \& Soter 1966; Matsumura et al. 2010; Barnes et al. 2011; Wilkins et al. 2017). In the following, we will use the modified tidal dissipation quality factor, $Q_{\star}^{\prime}$, defined by Ogilvie \& Lin (2007) as $Q_{\star}^{\prime} \equiv 1.5 Q_{\star} / k_{2}$, where $Q_{\star}$ is the stellar tidal quality factor and $k_{2}$ is the Love number for the second-order harmonic potential (Love 1944), which is related to the star's density profile.

The rate of the energy dissipation is related to the physical and orbital parameters of the planet as well as the spectral class, metallicity and rotational evolution of the parent star (Barker \& Ogilvie 2010; Ogilvie 2014; Essick \& Weinberg 2016; Gallet et al. 2017; Bolmont et al. 2017). Therefore, the orbital-decay time of a hot Jupiter is very difficult to estimate, but it should occur on a very long timescale (Rasio et al. 1996; Lai 2012), with evidence that the tidal destruction of hot Jupiters happens during the main-sequence lifetimes of their host stars (Chernov 2017; Hamer \& Schlaufman 2019).

Nevertheless, it is useful to try to determine the value of $Q_{\star}^{\prime}$, since it would provide insights into the interior parameters of the star and be an empirical test to current dynamical models of close-in planets and tidal stability. Theoretical studies of tidal evolution of hot Jupiters, based on different initial conditions and assumptions, suggest $10^{7}<Q_{\star}^{\prime}<10^{10}$ (Ogilvie 2014), $10^{6}<Q_{\star}^{\prime}<10^{9}$ (Penev \& Sasselov 2011; Penev et al. 2012) or $Q_{\star}^{\prime} \lesssim 10^{7}$ (Hamer \& Schlaufman 2019), A statistical inference based on a large sample of hot Jupiters yielded $Q_{\star}^{\prime} \sim 10^{8}$ (Collier Cameron \& Jardine 2018). Instead, Jackson et al. (2008) found that the distribution of initial eccentricities of close-in planet $(a<0.2 \mathrm{au})$ matches that of the general population, the best agreement being with $Q_{\star}^{\prime} \simeq 10^{5.5}$ A similar value of the tidal dissipation quality factor $\left(Q_{\star}^{\prime} \simeq 10^{5}-10^{6}\right)$ was estimated by Essick \& Weinberg (2016) for systems with solar-type host star, planet mass $M_{\mathrm{p}} \gtrsim 0.5 M_{\mathrm{Jup}}$ and orbital period $P \lesssim 2$ days. Therefore, such a planet can decay on smaller timescales than the mainsequence lifetime of its host.

\subsection{Transit timing variations for estimating $Q_{\star}^{\prime}$}

Under several assumptions and using Kepler's third law (Goldreich \& Soter 1966; Levrard et al. 2009; Matsumura et al. 2010; Birkby et al. 2014; Collier Cameron \& Jardine 2018), one can deduce that the tidal dissipation quality factor is related to the time derivative $\dot{P}$ of the planet's orbital period through

$Q_{\star}^{\prime}=-\frac{27 \pi}{2 \dot{P}} \frac{M_{\mathrm{p}}}{M_{\star}}\left(\frac{R_{\star}}{a}\right)^{5}$,

where $M_{\mathrm{p}}$ is the mass of the orbiting planet, $a$ is the semi-major axis of the orbit, while $M_{\star}$ and $R_{\star}$ are the mass and the radius of the parent star, respectively.

Variations of the orbital period can be found for those hot Jupiters that transit in front of their parent stars. The methodology is to systematically measure their mid-transit times and search for the so-called transit timing variations (TTVs). Such measurements are not difficult to achieve, although special attention is required for certain aspects, such as the clocks connected with the telescopes, the fit of the light curves, combining measurements made with different instruments, etc.

After excluding all possible sources of uncertainty, possible detection of TTVs in a transiting planetary system can be explained by several scenarios like the presence of additional bodies in the system, an apsidal precession or an unstable orbit, the latter resulting from tidal forces generated by the parent star. The orbital decay results in a non-zero time derivative of the orbital period, which can be measured, for example, by fitting transit timings to a quadratic ephemeris or by detecting long-term deviations from the linear ephemeris.

Constraining $Q_{\star}^{\prime}$ is, therefore, possible for a single transiting planet, but not easy to achieve, as it usually requires systematic observations of planetary-transit events over many years and high-precision photometry. Indeed, even though many hot Jupiters have been extensively monitored during the last two decades, only a few and controversial detections of orbital decay have been claimed to date (e.g., Adams et al. 2010; Murgas et al. 2014; Blecic et al. 2014; Hoyer et al. 2016; Jiang et al. 2016; Pablo et al. 2017; Southworth et al. 2019). Instead, Maciejewski et al. (2018b) found no departure from a constant-period model for WASP-18 b, despite this being one of the best candidates.

The most convincing case for the detection of orbital decay is that of the hot Jupiter WASP-12 b (Hebb et al. 2009), whose transits were systematically monitored almost immediately after the announcement of its discovery, straightway highlighting evidence of a decreasing orbital period (Maciejewski et al. 2013, 2016), which was later confirmed (Patra et al. 2017; Maciejewski et al. 2018a) and recently ascribed to the orbital decay of the planet (Yee et al. 2020; Turner et al. 2021). In particular, Turner et al. (2021) estimated for WASP-12 that $Q_{\star}^{\prime}=1.39 \pm 0.15 \times 10^{5}$, a low value if compared with most of the theoretical predictions, but in agreement with the previous estimate, $Q_{\star}^{\prime}=1.6 \pm 0.2 \times 10^{5}$, from Patra et al. (2020).

\subsection{Planets that may incur in orbital decay}

Considering Eq. (1), it is possible to appreciate how much WASP$12 \mathrm{~b}$ is a very good candidates for detecting orbital decay, as we can also see from Fig. 1, where we plotted $M_{\mathrm{p}} / M_{\star}$ versus $a / R_{\star}$ for the known transiting planets ${ }^{2}$. Another worthwhile plot to understand the impact of tidal interactions on planetary orbits is the tidal diagram (Pont et al. 2011). The circularisation time $\tau_{\mathrm{e}}$ can be written

2 Values taken from the Transiting Extrasolar Planet Catalogue (TEPCat), which is available at http://www.astro.keele.ac.uk/jkt/tepcat/ (Southworth 2011). 


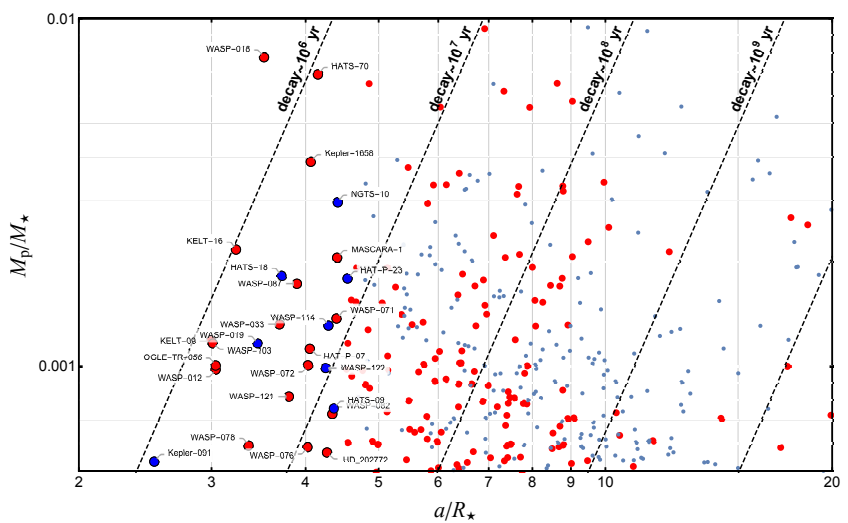

Figure 1. $M_{\mathrm{p}} / M_{\star}$ versus $a / R_{\star}$ diagram for the known transiting planetary systems (the values of the parameters were taken from TEPCat). The points representing the systems are marked with two different colours, according to the temperature of the host stars, i.e. red for those with $T_{\text {eff }}>6000 \mathrm{~K}$ and blue for the others. Error bars have been suppressed for clarity. Dark lines show where the decay timescale is constant and have been calculated using Eq. (1), considering a nominal value of $Q_{\star}^{\prime}=10^{6}$. The first twenty most favourable targets are highlighted and labelled. Figure inspired by Collier Cameron \& Jardine (2018) and Patra et al. (2020).

as (Matsumura et al. 2008; Bonomo et al. 2017) ${ }^{3}$

$\tau_{\mathrm{e}}=P_{\mathrm{orb}} \frac{M_{\mathrm{p}}}{M_{\star}} \frac{2 Q_{\mathrm{p}}^{\prime}}{63 \pi}\left(\frac{a}{R_{\mathrm{p}}}\right)^{5}$,

where $R_{\mathrm{p}}$ is the radius of the planet and $Q_{\mathrm{p}}^{\prime}$ is the planetary modified tidal quality factor, which is defined as $Q_{\mathrm{p}}^{\prime}=3 Q_{\mathrm{p}} / 2 k_{2}, Q_{\mathrm{p}}$ being the planet tidal quality factor. Following Bonomo et al. (2017), we considered a modified tidal diagram by plotting $P_{\text {orb }}\left(M_{\mathrm{p}} / M_{\star}\right)$ versus $\left(a / R_{\mathrm{p}}\right)$ in the top panel of Fig. 2 , so that the circularisation isochrones do not depend on the planetary orbital periods. From this plot, it is possible to appreciate how most of the eccentric planets, with $e>0.1$ (black stars), are found beyond the $1 \mathrm{Gyr}$ circularisation isochrone. Finally, in the bottom panel of Fig. 2 we show the transiting hot Jupiters with the smallest ratio $a / a_{\mathrm{R}}$.

Examining Figs. 1 and 2, KELT-16 b is one of the most promising hot Jupiters whose transits are worth to precisely record for searching possible tidal-decay signatures. This planet is the object we selected for the study that we present in this work and its properties are described in Sect. 2. The paper is organized as follows. In Sect. 3 we present new photometric follow-up observations of KELT-16 b transits. The data reduction is also described in Sect. 3. The analysis of the light curves is presented in Sect. 4, while, in Sect. 5, we analysed the transit times and investigated the possibility of a decay of the planetary orbit/In Sect. 6 we revise the main physical properties of the KELT-16 planetary system and investigated the atmosphere composition of its planet. Finally, we summarise our results in Sect. 7 .

\section{THE CASE STUDY: KELT-16 B}

KELT-16 b was discovered by Oberst et al. (2017). It has $M_{\mathrm{p}}=$ $2.71 M_{\text {Jup }}, R_{\mathrm{p}}=1.38 R_{\text {Jup }}$ and orbits in 0.97 days at $\approx 1.8 \mathrm{a} / a_{\mathrm{R}}$ around the bright $(V=11.7 \mathrm{mag})$ F7V star KELT-16 (aka

3 Note that the $\pi$ in Eq. (1) of Bonomo et al. 2017 was incorrectly reversed, as confirmed by Bonomo (2021), private communication.
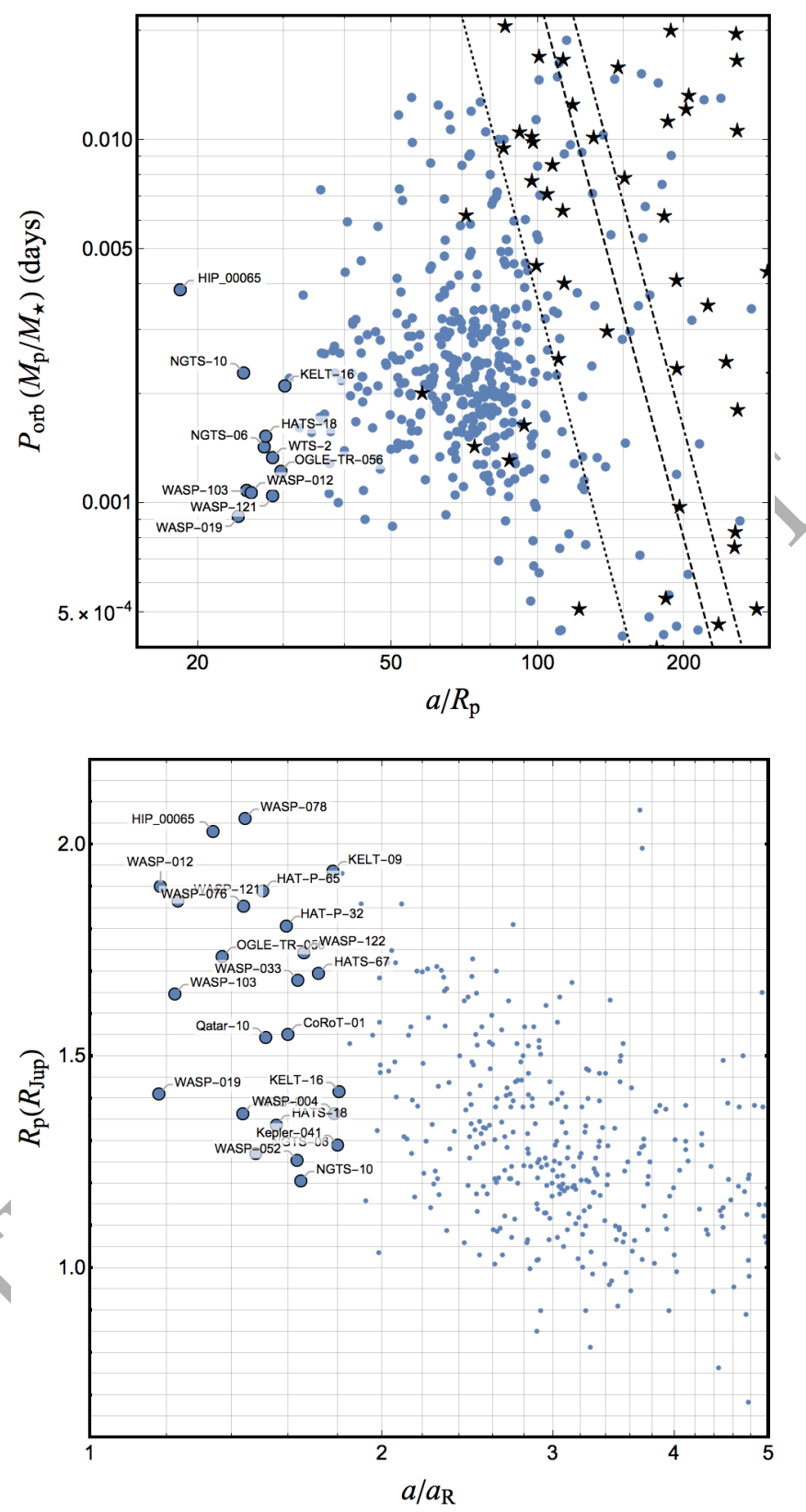

Figure 2. Top panel: Modified tidal diagram of known transiting exoplanets. Circles display the position of planets with eccentricity $e<0.1$, while five-pointed stars those with $e>0.1$. Error bars have been suppressed for clarity. The dotted, dashed and dash-dotted lines display the 1,7 , and $14 \mathrm{Gyr}$ circularisation timescales for $Q_{\mathrm{p}}^{\prime}=10^{6}$ and $e=0$, respectively. Figure inspired from Bonomo et al. (2017). Bottom panel: Distribution of the known larger transiting hot Jupiters versus the orbital semi-major axis in units of Roche radii $a_{\mathrm{R}}$. The values of the parameters were taken from TEPCat. The planets close to tidal disruption are highlighted and labelled.

TYC 2688-1839-1, aka TOI-1282) in the thin disk of the Galaxy, on the outskirts of the Cygnus Loop nebula, a supernova remnant. This star has a widely separated bound companion (a $V=19.6$ mag, M3 $\mathrm{V}$ type red dwarf), with a minimum separation of $\approx 300 \mathrm{au}$ and an effective temperature of $T_{\text {eff }} \approx 3400 \mathrm{~K}$ (Oberst et al. 2017). This suggests the possibility to have Kozai-Lidov (KL) oscillations, which could be responsible for having driven KELT-16 b to its current orbit. 


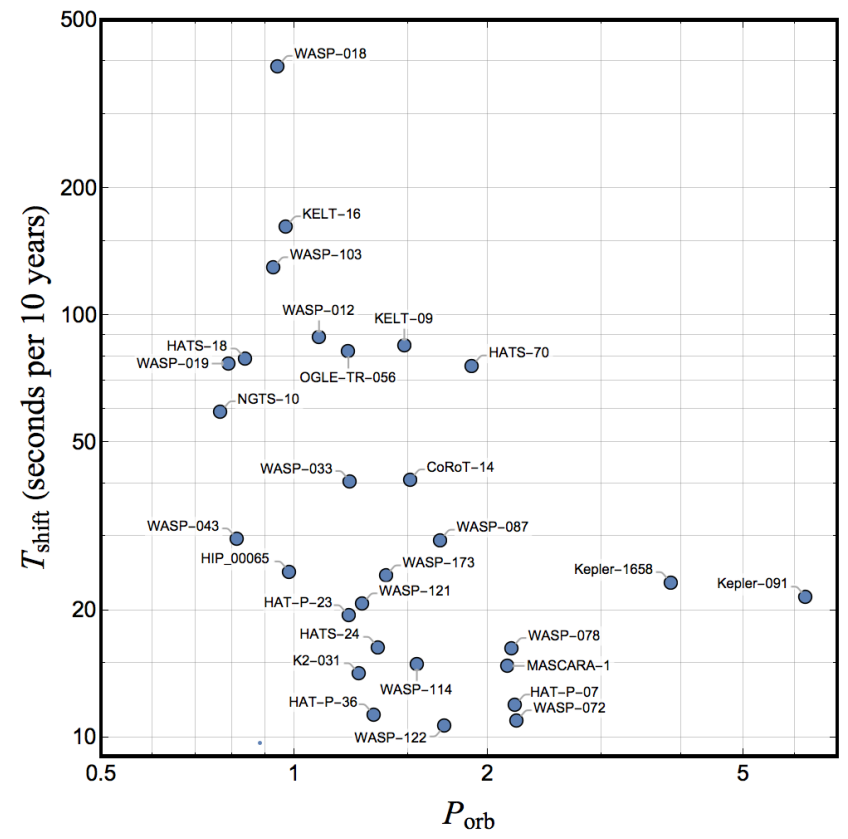

Figure 3. $T_{\text {shift }}-P_{\text {orb }}$ diagram for the known transiting hot Jupiters, whose transits should present the largest mid-transit time shifts after $10 \mathrm{yr}$. These planets are labelled. Data taken from TEPCat. Figure inspired from Maciejewski et al. (2018a).

With an equilibrium temperature of $\approx 2450 \mathrm{~K}$, KELT- $16 \mathrm{~b}$ can be considered as an ultra-hot Jupiter. By using an orbital evolution model, Oberst et al. (2017) showed that KELT-16 b could be tidally disrupted in roughly $5.5 \times 10^{5} \mathrm{yr}$, if its parent stars has a value of $Q_{\star}^{\prime} \sim 10^{5}$, i.e. similar to that of WASP-12, which was mentioned above (see Sect. 1).

Due to its particular orbital period $(\approx 1$ day), there are several blocks of consecutive nights in which KELT-16 b undergoes transit events during a year. This is a rare and peculiar characteristic for a transiting planet, because it allows intensive and continuous monitoring of planetary-transit times for all these nights with groundbased telescopes, also simplifying the schedule of observations.

Maciejewski et al. (2018a) selected this target as one of the best candidates in the northern hemisphere for detecting planet-star tidal interactions. Their selection included planets for which the $T_{\text {shift }}$, the predicted cumulative shift in transit times, was greater than $30 \mathrm{~s}$ after 10 years. Figure 3 shows the $T_{\text {shift }}-P_{\text {orb }}$ diagram for transiting hot Jupiters, where $T_{\text {shift }}$ was derived from Eq. (1) (see also Maciejewski et al. 2018a), in which the position of KELT-16 b, as well as those of other hot Jupiters, is highlighted.

Eleven transit observations were reported by Maciejewski et al. (2018a), who used an array of five different telescopes, with apertures between $0.6 \mathrm{~m}$ and $2 \mathrm{~m}$. The corresponding mid-transit times were joined with those from the discovery paper (Oberst et al. 2017), finding that they were well fitted by a linear ephemeris. The same result was obtained by Patra et al. (2020), who observed another two transits of KELT-16 b with a $1.2 \mathrm{~m}$ telescope.

Finally, a Spitzer/IRAC $4.5 \mu \mathrm{m}$ phase curve was reported by Bell et al. (2021), who estimated the day- and night-side temperature of the planet.

\section{OBSERVATIONS AND DATA REDUCTION}

Starting from summer 2017, we used an array of five different telescopes (see Table 1) to perform photometric follow-up observations of KELT-16. In particular, we used the Zeiss 1.23 m telescope at Calar Alto (Spain), the Cassini $1.52 \mathrm{~m}$ telescope at the INAFOAS Observatory (Italy), the T100 $1.0 \mathrm{~m}$ telescope at the TUG Observatory (Turkey), the SPM $84 \mathrm{~cm}$ telescope at the National Astronomical Observatory (Mexico) and the $80 \mathrm{~cm}$ telescope at the OARPAF Observatory (Italy). The observations were carried out through different optical passbands (covering 400-1000 nm) to also study the variation of the planetary radius with the wavelength (Southworth et al. 2012b), see Sect. 6.3.

We recorded 30 planetary transits ${ }^{4}$, including three transits that were simultaneously observed with two telescopes (Cassini and CA) and one with three telescopes (Cassini, CA and OARPAF), obtaining a total of 36 light curves (Figs. 4, 5, 6, 7).

Observations of a planetary-transit event by multiple telescopes from different places are not easy to achieve since one needs the same telescope time at two different observatories and the weather conditions must be favourable at both observation sites. If both conditions are met, the corresponding light curves are useful to better constrain the contact points and, therefore, the mid-transit time, as well as unambiguously understand if any feature present in the light curve is due to a real astrophysical signal (e.g., starspot) or systematic noise (Ciceri et al. 2013; Mancini et al. 2013a, 2017).

The light curves obtained from our simultaneous observations are plotted superimposed in Appendix A.

\subsection{CA $1.23 \mathrm{~m}$ telescope}

Fourteen complete transits of KELT-16b were observed with the Zeiss $1.23 \mathrm{~m}$ telescope at the German-Spanish Astronomical Center at Calar Alto (CA) in Spain. The telescope is equipped with a DLRMKIII $4 \mathrm{k} \times 4 \mathrm{k}$ camera, which has pixels of size $15 \mu \mathrm{m}$. Considering that the focal length of the telescope is $9857.1 \mathrm{~mm}$, the resulting field-of-view (FOV) is $21.5 \times 21.5 \mathrm{arcmin}$, which guarantees a good number of comparison stars in almost all cases. Observations were all performed using autoguiding and the defocussing method, in order to increase the photometric precision (Southworth et al. 2009). The CCD was windowed to decrease the readout time and increase the cadence of the observations. The transits were observed through standard Johnson $B, V$ filters and Cousins $R I$ filters. Details of the observations are reported in Table 1.

The data were analysed in the standard way by using the IDL/DEFOT pipeline (Southworth et al. 2009). In short, we calibrated the raw images of the target with masterbias and masterflat frames, which were obtained by median combining a set of individual bias and sky flat-field images, taken on the same day as each transit observation. We corrected pointing variations by cross-correlating each image against a reference frame, selected considering the airmass. Aperture photometry was performed by placing three apertures on the target and comparison stars. The sizes of the concentric apertures were selected based on the lowest scatter obtained when compared with a fitted model (see Table 1). Differential-magnitude light curves were generated for each observing sequence versus an ensemble of comparison stars. A straight line was fitted to the observations outside transit and subtracted to normalize the final light

4 The reduced data will be made available at the cDs catalogue service, http://cdsweb.u-strasbg.fr/ 
Table 1. Details of the transit observations presented in this work. $N_{\mathrm{obs}}$ is the number of observations, $T_{\mathrm{exp}}$ is the exposure time, $T_{\mathrm{obs}}$ is the observational cadence, and 'Moon illum.' is the geocentric fractional illumination of the Moon at midnight (UT). The aperture sizes are the radii of the software apertures for the star, inner sky and outer sky, respectively. Scatter is the rms scatter of the data versus a fitted model. The last column specifies if the transit was simultaneously observed by more than one telescope.

\begin{tabular}{|c|c|c|c|c|c|c|c|c|c|c|c|c|}
\hline Telescope & $\begin{array}{l}\text { Date of } \\
\text { first obs }\end{array}$ & $\begin{array}{l}\text { Start time } \\
\text { (UT) }\end{array}$ & $\begin{array}{l}\text { End time } \\
\text { (UT) }\end{array}$ & $N_{\text {obs }}$ & $\begin{array}{l}T_{\exp } \\
(\mathrm{s})\end{array}$ & $\begin{array}{c}T_{\mathrm{obs}} \\
(\mathrm{s})\end{array}$ & Filter & Airmass & $\begin{array}{l}\text { Moon } \\
\text { illum. }\end{array}$ & $\begin{array}{c}\text { Aperture } \\
\text { radii }(\mathrm{px})\end{array}$ & $\begin{array}{l}\text { Scatter } \\
\text { (mmag) }\end{array}$ & Simultaneous \\
\hline CA $1.23 \mathrm{~m}$ & 2017/06/19 & $23: 58$ & 03:46 & 191 & 60 & 72 & Cousins $I$ & $1.31 \rightarrow 1.00 \rightarrow 1.01$ & $22 \%$ & $16,25,49$ & 1.27 & no \\
\hline CA $1.23 \mathrm{~m}$ & $2017 / 06 / 20$ & $21: 59$ & $03: 50$ & 295 & 60 & 72 & Cousins $I$ & $2.21 \rightarrow 1.00 \rightarrow 1.01$ & $14 \%$ & $17,26,48$ & 1.67 & no \\
\hline Cassini $1.52 \mathrm{~m}$ & $2017 / 06 / 21$ & $21: 56$ & $02: 41$ & 235 & $40 / 95$ & $58 / 113$ & Johnson $R$ & $1.33 \rightarrow 1.02 \rightarrow 1.06$ & $7 \%$ & $11,15,23$ & 1.60 & yes \\
\hline $\mathrm{CA} 1.23 \mathrm{~m}$ & $2017 / 06 / 21$ & $22: 12$ & $04: 11$ & 230 & $60 / 95$ & $72 / 107$ & Cousins $R$ & $1.95 \rightarrow 1.00 \rightarrow 1.04$ & $7 \%$ & $18,27,49$ & 1.21 & yes \\
\hline OARPAF $80 \mathrm{~cm}$ & $2017 / 06 / 21$ & $22: 18$ & $02: 23$ & 225 & 50 & 62 & Johnson $R$ & $1.03 \rightarrow 1.29$ & $7 \%$ & $14,15,24$ & 2.15 & yes \\
\hline $\mathrm{CA} 1.23 \mathrm{~m}$ & $2017 / 06 / 22$ & $21: 42$ & 03:00 & 232 & $55 / 95$ & $67 / 107$ & Cousins $R$ & $2.15 \rightarrow 1.00$ & $2 \%$ & $18,26,49$ & 1.60 & yes \\
\hline Cassini $1.52 \mathrm{~m}$ & $2017 / 06 / 22$ & $22: 18$ & $02: 36$ & 230 & $40 / 75$ & $58 / 93$ & Johnson $R$ & $1.36 \rightarrow 1.02 \rightarrow 1.07$ & $2 \%$ & $12,16,25$ & 1.25 & yes \\
\hline Cassini $1.52 \mathrm{~m}$ & $2017 / 06 / 23$ & $22: 00$ & $01: 15$ & 134 & $40 / 90$ & $58 / 108$ & Johnson $V$ & $1.40 \rightarrow 1.02$ & $0 \%$ & $11,14,25$ & 2.00 & no \\
\hline $\mathrm{T} 1001 \mathrm{~m}$ & $2017 / 06 / 25$ & $19: 37$ & $01: 16$ & 126 & 110 & 155 & Bessel $R$ & $2.17 \rightarrow 1.01$ & $5 \%$ & $15,20,25$ & 1.82 & no \\
\hline SPM $84 \mathrm{~cm}$ & $2017 / 07 / 10$ & $06: 43$ & $12: 01$ & 108 & 150 & 162 & Bessel $R$ & $1.22 \rightarrow 1.00 \rightarrow 1.18$ & $95 \%$ & $20,22,27$ & 2.40 & no \\
\hline $\mathrm{SPM} 84 \mathrm{~cm}$ & $2017 / 07 / 11$ & $05: 57$ & $11: 58$ & 135 & 150 & 162 & Bessel $R$ & $1.39 \rightarrow 1.00 \rightarrow 1.18$ & $89 \%$ & $20,22,27$ & 1.35 & \\
\hline Cassini $1.52 \mathrm{~m}$ & $2017 / 07 / 22$ & $21: 56$ & $02: 41$ & 269 & $40 / 95$ & $58 / 113$ & Johnson $V$ & $1.33 \rightarrow 1.02 \rightarrow 1.06$ & $0 \%$ & $11,15,23$ & 1.60 & \\
\hline Cassini $1.52 \mathrm{~m}$ & $2017 / 07 / 24$ & $20: 51$ & 02:07 & 113 & $120 / 135$ & $138 / 153$ & Johnson $B$ & $1.26 \rightarrow 1.02 \rightarrow 1.24$ & $4 \%$ & $15,20,50$ & 1.16 & \\
\hline Cassini $1.52 \mathrm{~m}$ & $2017 / 07 / 25$ & $20: 11$ & $01: 36$ & 163 & $95 / 120$ & $113 / 138$ & Johnson $B$ & $1.32 \rightarrow 1.02 \rightarrow 1.36$ & $9 \%$ & $13,20,52$ & & no \\
\hline $\mathrm{T} 1001 \mathrm{~m}$ & $2017 / 07 / 27$ & $18: 17$ & $22: 19$ & 92 & 110 & 155 & Bessel I & $1.67 \rightarrow 1.01$ & $24 \%$ & $30,40,45$ & 1.44 & no \\
\hline $\mathrm{CA} 1.23 \mathrm{~m}$ & $2017 / 08 / 20$ & $22: 52$ & $04: 38$ & 287 & 60 & 72 & Cousins $I$ & $1.05 \rightarrow 1.00 \rightarrow 1.68$ & $1 \%$ & $18,28,47$ & & no \\
\hline $\mathrm{CA} 1.23 \mathrm{~m}$ & $2017 / 08 / 22$ & $21: 19$ & $04: 23$ & 351 & 60 & 72 & Cousins $R$ & $1.11 \rightarrow 1.00 \rightarrow 2.23$ & $2 \%$ & $18,30,50$ & 1.45 & no \\
\hline $\mathrm{CA} 1.23 \mathrm{~m}$ & $2017 / 08 / 23$ & $21: 02$ & $02: 53$ & 295 & 60 & 72 & Johnson $V$ & $1.35 \rightarrow 1.00 \rightarrow 1.93$ & $6 \%$ & $18,28,47$ & 1.53 & no \\
\hline $\mathrm{CA} 1.23 \mathrm{~m}$ & $2017 / 09 / 24$ & $20: 21$ & $02: 33$ & 192 & 100 & 112 & Johnson $V$ & $1.01 \rightarrow 1.00 \rightarrow 2.58$ & $23 \%$ & $15,24,50$ & 1.34 & no \\
\hline $\mathrm{CA} 1.23 \mathrm{~m}$ & $2017 / 09 / 25$ & $19: 15$ & 02:00 & 174 & $100 / 180$ & $112 / 192$ & Johnson $B$ & $1.07 \rightarrow 1.00 \rightarrow 2.12$ & $31 \%$ & $22,32,55$ & 1.55 & no \\
\hline $\mathrm{CA} 1.23 \mathrm{~m}$ & $2017 / 09 / 26$ & $19: 28$ & $01: 22$ & 284 & $50 / 100$ & $62 / 112$ & Cousins $R$ & $1.04 \rightarrow 1.00 \rightarrow 1.76$ & $40 \%$ & $16,25,50$ & 1.87 & no \\
\hline $\mathrm{CA} 1.23 \mathrm{~m}$ & $2017 / 09 / 29$ & $18: 00$ & $04: 35$ & 226 & $55 / 100$ & $67 / 112$ & Cousins $R$ & $1.01 \rightarrow 1.00 \rightarrow 2.42$ & $68 \%$ & $16,26,50$ & 1.11 & no \\
\hline CA $1.23 \mathrm{~m}$ & $2018 / 07 / 01$ & $22: 19$ & $02: 16$ & 136 & $90 / 120$ & $102 / 132$ & Cousins $I$ & $1.80 \rightarrow 1.00 \rightarrow 1.03$ & $87 \%$ & $12,35,55$ & 1.06 & no \\
\hline $\mathrm{CA} 1.23 \mathrm{~m}$ & $2018 / 07 / 02$ & $21: 43$ & $03: 17$ & 139 & 120 & 132 & Cousins $R$ & $1.81 \rightarrow 1.00 \rightarrow 1.02$ & $80 \%$ & $18,27,47$ & 1.19 & yes \\
\hline Cassini $1.52 \mathrm{~m}$ & $2018 / 07 / 02$ & $21: 23$ & $02: 42$ & 122 & 120 & 138 & Johnson $R$ & $1.32 \rightarrow 1.02 \rightarrow 1.12$ & $81 \%$ & $14,18,28$ & 1.33 & yes \\
\hline Cassini $1.52 \mathrm{~m}$ & $2018 / 07 / 03$ & $21: 20$ & $23: 56$ & 70 & 120 & 138 & Johnson $V$ & $1.31 \rightarrow 1.03$ & $72 \%$ & $12,18,25$ & 2.10 & yes \\
\hline CA $1.23 \mathrm{~m}$ & $2018 / 07 / 03$ & $21: 39$ & $02: 25$ & 121 & $120 / 130$ & $132 / 142$ & Cousins $R$ & $1.87 \rightarrow 1.00$ & $72 \%$ & $22,30,47$ & 1.22 & yes \\
\hline $\mathrm{T} 1001 \mathrm{~m}$ & $2019 / 07 / 12$ & $22: 03$ & 01:20 & 79 & 90 & 135 & Bessel $R$ & $1.06 \rightarrow 1.00 \rightarrow 1.07$ & $85 \%$ & $20,25,30$ & 1.79 & no \\
\hline $\mathrm{T} 1001 \mathrm{~m}$ & $2019 / 08 / 14$ & $20: 03$ & $00: 33$ & 303 & 30 & 45 & Bessel $R$ & $1.05 \rightarrow 1.00 \rightarrow 1.28$ & $96 \%$ & $13,21,26$ & 7.13 & no \\
\hline $\mathrm{T} 1001 \mathrm{~m}$ & $2019 / 09 / 16$ & $18: 50$ & 23:07 & 297 & 30 & 45 & Bessel $R$ & $1.01 \rightarrow 1.00 \rightarrow 1.48$ & $93 \%$ & $10,17,22$ & 3.78 & no \\
\hline $\mathrm{T} 1001 \mathrm{~m}$ & $2019 / 09 / 17$ & $17: 16$ & $23: 05$ & 422 & 30 & 45 & Bessel $R$ & $1.10 \rightarrow 1.00 \rightarrow 1.49$ & $87 \%$ & $12,17,22$ & 2.88 & no \\
\hline $\mathrm{T} 1001 \mathrm{~m}$ & $2020 / 05 / 20$ & $21: 26$ & $01: 42$ & 85 & 110 & 155 & Bessel $R$ & $2.55 \rightarrow 1.05$ & $3 \%$ & $17,33,46$ & 1.73 & no \\
\hline Cassini $1.52 \mathrm{~m}$ & $2020 / 07 / 19$ & $20: 26$ & $02: 28$ & 263 & 120 & 138 & GG-495 & $1.31 \leadsto 1.04$ & $1 \%$ & $12,18,25$ & 1.03 & no \\
\hline $\mathrm{T} 1001 \mathrm{~m}$ & $2021 / 01 / 03$ & $15: 40$ & $17: 47$ & 43 & 120 & 165 & Bessel $R$ & $1.42 \rightarrow 2.82$ & $76 \%$ & $14,24,33$ & 1.90 & no \\
\hline Cassini $1.52 \mathrm{~m}$ & $2021 / 06 / 29$ & $21: 15$ & $02: 21$ & 187 & 60 & 78 & Johnson $V$ & $1.70 \rightarrow 1.03 \rightarrow 1.05$ & $68 \%$ & $10,30,55$ & 1.83 & no \\
\hline Cassini $1.52 \mathrm{~m}$ & $2021 / 06 / 30$ & $22: 06$ & $02: 17$ & 181 & 60 & 78 & Johnson $V$ & $1.35 \rightarrow 1.03 \rightarrow 1.05$ & $58 \%$ & $10,30,50$ & 1.08 & no \\
\hline
\end{tabular}

curves to zero differential magnitude. The weights of the comparison stars were simultaneously optimized to minimize the scatter in the data points outside transits. Since the aperture-photometry procedure tends to underestimate the uncertainties of the measurements, they were then scaled so each transit had a reduced $\chi^{2}$ of $\chi_{v}^{2}=1.0$ versus a best-fitting model calculated with the JKTEBOP code (see Sect. 4). The final light curves are plotted in Fig. 4. Unfortunately, the suboptimal quality of the data is due to the fact that there is only one good comparison star (TYC 2688-1883-1) in the FOV. This limits the photometric accuracy, even using defocussing, when compared with other transiting systems with similar magnitude, where we have many more comparison stars and we usually obtain lower scatter $(<1 \mathrm{mmag})$ per observation.

\subsection{Cassini $1.52 \mathrm{~m}$ telescope}

Eleven transits of KELT-16 b were observed with the Cassini $1.52 \mathrm{~m}$ telescope from the Astrophysics and Space Science Observatory of Bologna in Loiano (Italy) by using the BFOSC (Bologna Faint Object Spectrograph and Camera). This imager has a back-illuminated CCD with $1300 \times 1340$ pixels and a pixel size of $20 \mu \mathrm{m}$. The current FOV of this facility is $13 \times 12.6$ arcmin. The transits were observed through standard Johnson $B, V, R$ filters and one with the special uncoated GG-495 glass long-pass filter (transparent at $>500 \mathrm{~nm}$ ), which is used within the EDEN project (Gibbs et al. 2020). Sev- eral of these transits were, unfortunately, not well sampled due to bad weather conditions (see Fig. 5). Details of the observations are reported in Table 1. The data were reduced and analysed as those from CA (see Sect. 3.1).

\subsection{T100 $1 \mathrm{~m}$ telescope}

We observed eight transits of KELT-16 b with the 1-meter Turkish telescope T100 in TÜBİTAK National Observatory of Turkey's (TUG) Bakırlitepe Campus, which is located at $2500 \mathrm{~m}$ altitude. The telescope is equipped with a cryo-cooled SI 1100 CCD with $4096 \times 4096$ pixels, which gives an effective field of view of $20^{\prime} \times$ $20^{\prime}$. We employed a Bessel- $R$ filter in all the observations except that on 2017/07/27, in which a Bessel- $I$ filter was used. We employed the defocusing technique in 5 nights, while we focused the telescope during the nights of August 14, September 16-17 in 2019. Details of the observations are reported in Table 1. The AstroImagEJ software package was used (Collins et al. 2017) for reducing the CCD images and performing aperture photometry with respect an ensemble of comparison stars. The light curves are plotted in Fig. 6.

\subsection{SPM $84 \mathrm{~cm}$ telescope}

Two complete transits of KELT-16 b were observed with the $84 \mathrm{~cm}$ telescope of the Observatorio Astronomico Nacional of San Pe- 

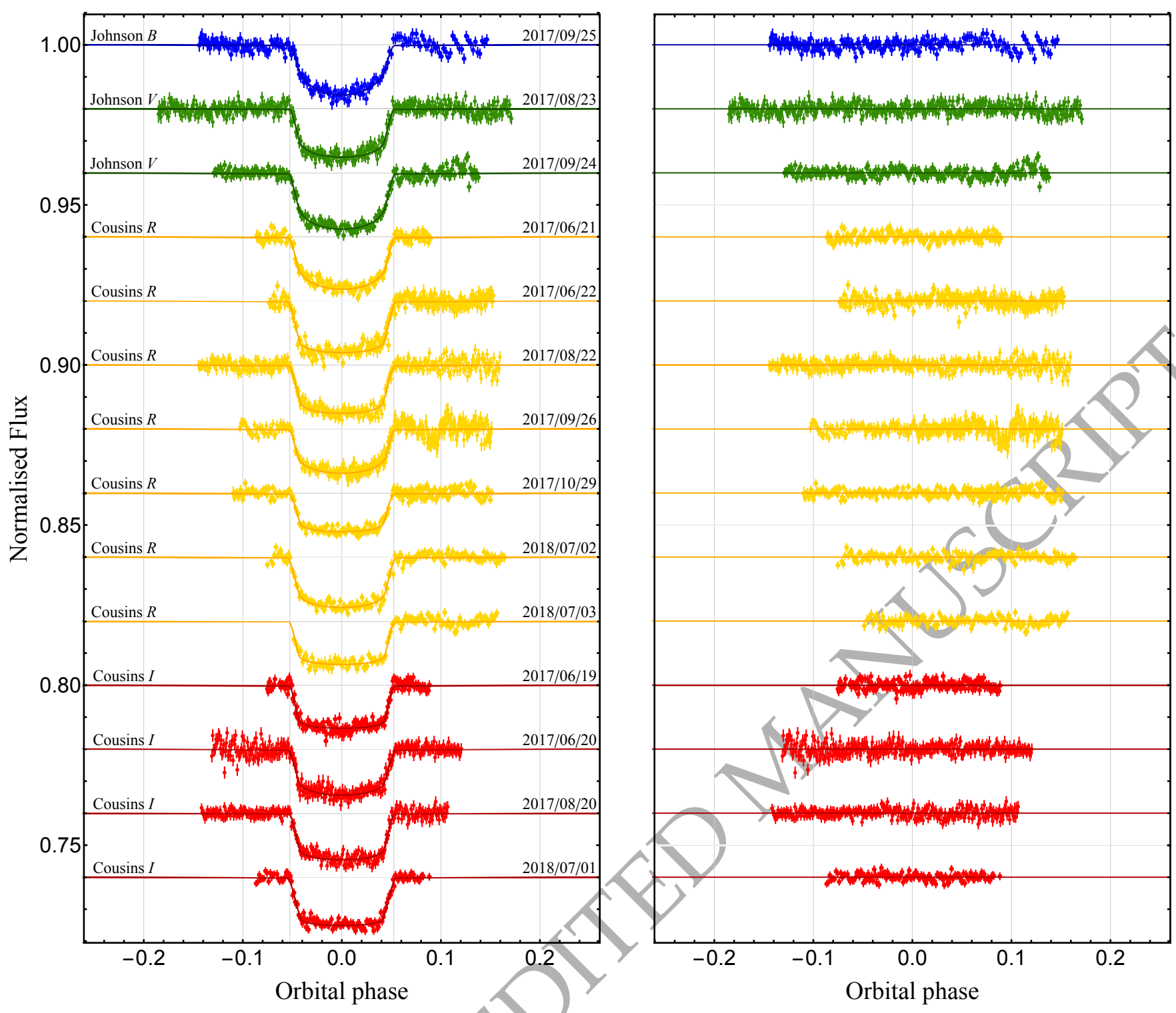

Figure 4. Left-hand panel: light curves of fourteen transits of KÊLT-16b observed with the Zeiss $1.23 \mathrm{~m}$ telescope through four different filters. The labels indicate the observation date and the filter that was used for each observation. They are plotted versus the orbital phase and compared to the best-fitting JKTEBOP models. Right-hand panel: the residuals of each fit.

dro Mártir (OAN-SPM), which is located in Northwestern Mexico. This telescope provides a set of instruments that can be mounted according to the observational needs; among these, we have used the Mexman, which is a wide-field imager with a CCD size of

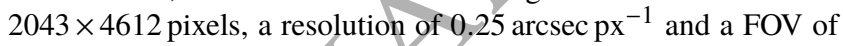
$8.4 \times 19.0$ arcmin. The transits were observed through a Bessel- $R$ filter and the photometric data were reduced and analysed as those from CA (see Fig. 7). Details of the observations are reported in Table 1, while those about this telescope can be found in Ricci et al. (2017).

\subsection{OARPAF $80 \mathrm{~cm}$ telescope}

One complete transit of KELT-16b was observed with the altazimuth OARPAF $80 \mathrm{~cm}$ telescope, located near Mt. Antola in Northern Italy. At the time of the observation, the telescope was equipped with an air-cooled SBIG STL $11000 \mathrm{~m}$ camera and a standard UBVRI Johnson filter wheel. The size of this CCD is $2004 \times 1336$ pixels, with a resolution of $0.29 \operatorname{arcsec} \mathrm{px}^{-1}$ and a FOV of $10 \times 10 \mathrm{arcmin}$. The transit was observed using the Johnson $R$ filter and the photometric data were reduced and analysed as those from CA (see Fig. 7). Details of the observations are reported in Table 1, while those about this telescope can be found in Ricci et al. (2020).

\subsection{TESS photomety}

KELT-16 was observed by TESS (Ricker et al. 2015) with the 2 min cadence during Sector 15 of its primary mission. Continuous observations of the target star were obtained from 201908-15 to 2019-08-25 and from 2019-08-29 to 2019-09-07. Using the Lightkurve Collaboration (2018) Python package, we downloaded the detrended PDCSAP (Pre-search Data Conditioning Simple Aperture Photometry) light curve, whose known systematics 

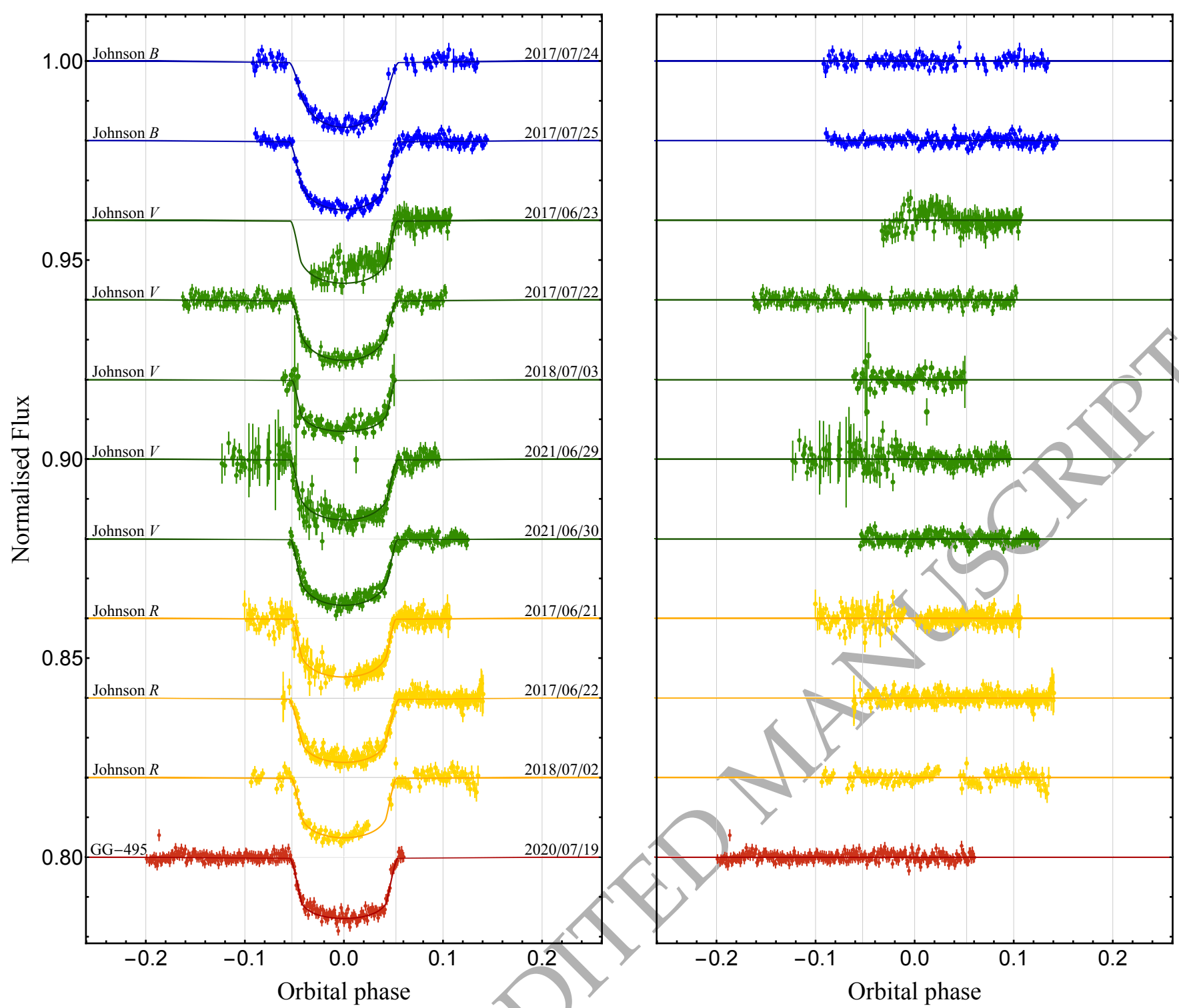

Figure 5. Left-hand panel: light curves of eight transits of KELT-16 b observed with the Cassini $1.52 \mathrm{~m}$ telescope through four different filters. The labels indicate the observation date and the filter that was used for each observation. They are plotted versus the orbital phase and compared to the best-fitting JKTEBOP models. Right-hand panel: the residuals of each fit.

were already removed. The data contains twenty transits of KELT$16 \mathrm{~b}$, of which eighteen are complete and present out-of-transit data both before the ingress and after the egress, see Fig. 8. The suboptimal quality of TESS data is due to the faintness of the KELT-16 $\operatorname{star}(V=11.6 \mathrm{mag})$, which is at the limit of the working magnitude of TESS.

\section{LIGHT-CURVE ANALYSIS}

First of all, we performed a careful analysis of the most suspicious light curves using PRISM and GEMC codes (Tregloan-Reed et al. 2013, 2015), in order to search for the possible presence of starspots occulted by KELT-16 b during its transits. It was reported by Tregloan-Reed \& Unda-Sanzana $(2019,2021)$ that to fully constrain the physical parameters of a starspot and therefore provide a detection to a high confidence, requires the amplitude of a starspot anomaly to be at least twice the rms scatter of the light curve. Our analysis determined that all the anomaly amplitudes were below this limit and when modelled as a starspot anomaly, gave inconsistent system parameters (i.e. transit depth) compared to the other anomaly free transits, strongly suggesting that the anomalies are non astrophysical systematics. The absence of starspots is a conclusion in agreement with a low stellar activity, which is typical for the spectral class of the parent star.

Then, we considered the possible light contribution coming from the companion M3 star (see Sect. 1.1), which is at roughly $0^{\prime \prime} .7$ away from KELT-16, but much fainter and cooler than it. Following Southworth et al. (2010), we estimated the amount of this "third light" by considering the effective temperature of the two stars, the $\Delta K$ mag measured by Oberst et al. (2017), the 2MASS $K_{\mathrm{S}}$ filter and extrapolated the light ratios to the $B, V, R, I$ bands using synthetic spectra, which were calculated with the ATLAS9 (Kurucz 1979, 1993) and PHOENIX model atmospheres (Allard et al. 2001), and filter-response functions taken from the Isaac Newton Group of 


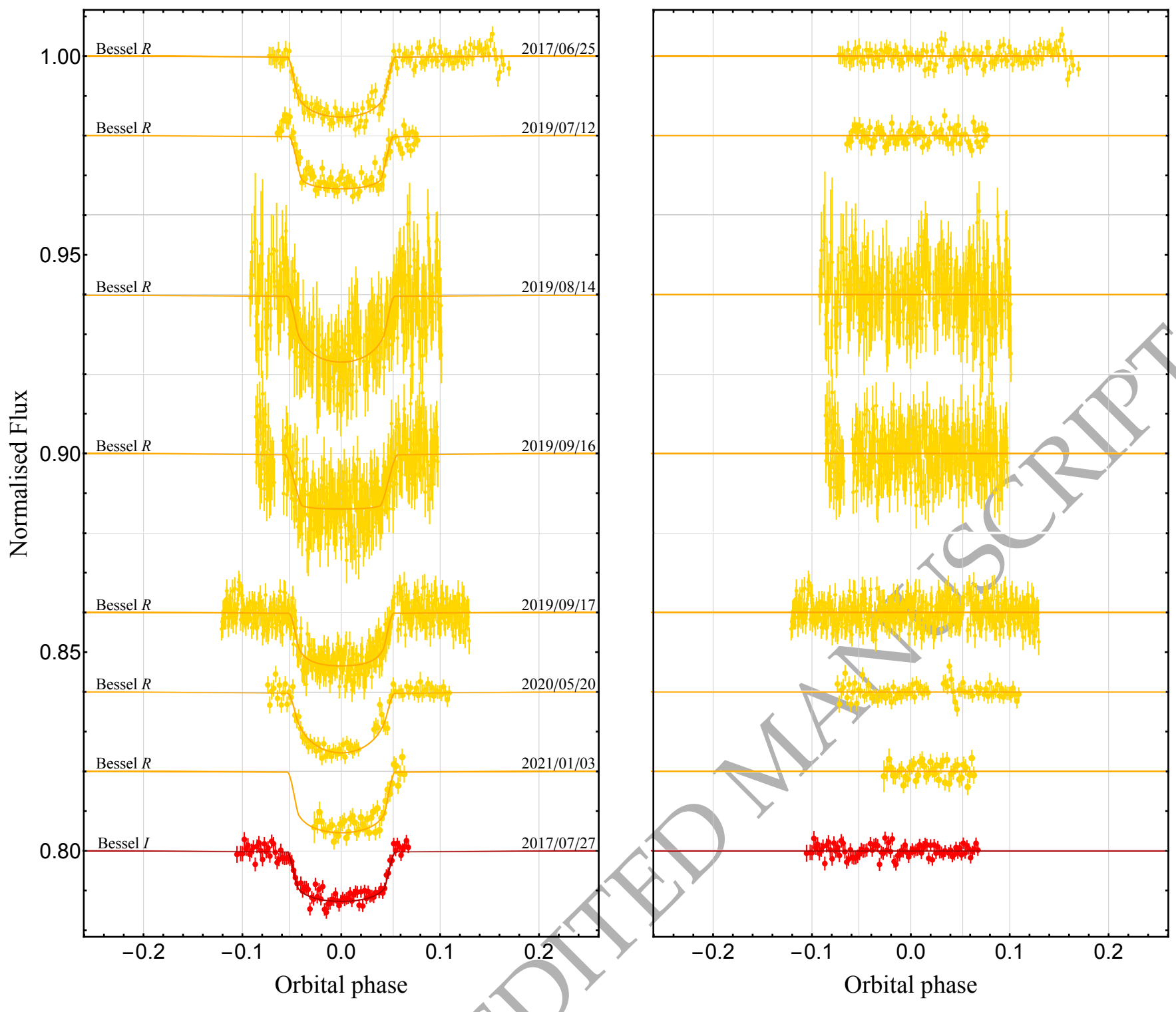

Figure 6. Left-hand panel: light curves of eight transits of KELT-16b observed with the T100 $1 \mathrm{~m}$ telescope through two different filters. The labels indicate the observation date and the filter that was used for each observation. They are plotted versus the orbital phase and compared to the best-fitting JKTEBOP models. Right-hand panel: the residuals of each fit.

Telescopes website ${ }^{5}$. We found the following values for the fraction of light coming from the unresolved star:

- Johnson $B: \quad 0.00035 \pm 0.00009$

- Johnson $V$ : $\quad 0.00076 \pm 0.00020$

- Johnson/Cousins $R: 0.00147 \pm 0.00037$

- Johnson/Cousins I: $0.00400 \pm 0.00095$

Therefore, in the worst case, the contribution of the companion is only $0.4 \%$. We made seyeral tests with our $I$-band light curves to see how much effect this is compared to the error bars and found that it does not make a significant difference for the global solution of the system (see Sect. 6).

As a next step, following our consolidated approach (see, for example, Mancini et al. 2014; Southworth et al. 2016 and reference therein), we used the Jктевор code (Southworth 2013) to model the

\footnotetext{
5 http://www.ing.iac.es.
}

light curves presented in Sect. 3. The orbit of the planet was assumed circular (Oberst et al. 2017) and the light curves were modelled using the Levenberg-Marquardt optimization algorithm. In particular, we fitted the sum and ratio of the fractional radii $^{6}\left(r_{\star}+r_{\mathrm{p}}\right.$ and $\left.k=r_{\mathrm{p}} / r_{\star}\right)$, the orbital period and inclination $\left(P_{\text {orb }}\right.$ and $\left.i\right)$ and the midpoint time of the transit $\left(T_{0}\right)$. The limb darkening (LD) of the star was also modelled by applying a quadratic law for describing the LD effect and using the LD coefficients provided by Claret et al. (2004). We considered the linear coefficient as a free parameter and fixed the non-linear one, which was perturbed during the error-analysis process. Finally, the uncertainties of the fitted parameters were estimated by running a residual-permutation algorithm (Southworth 2008).

The light curves and their best-fitting models are shown in 6 The fractional radii are $r_{\star}=R_{\star} / a$ and $r_{\mathrm{p}}=R_{\mathrm{p}} / a$, where $R_{\star}$ and $R_{\mathrm{p}}$ are
the true radii of the star and planet, while $a$ is the semi-major axis. 


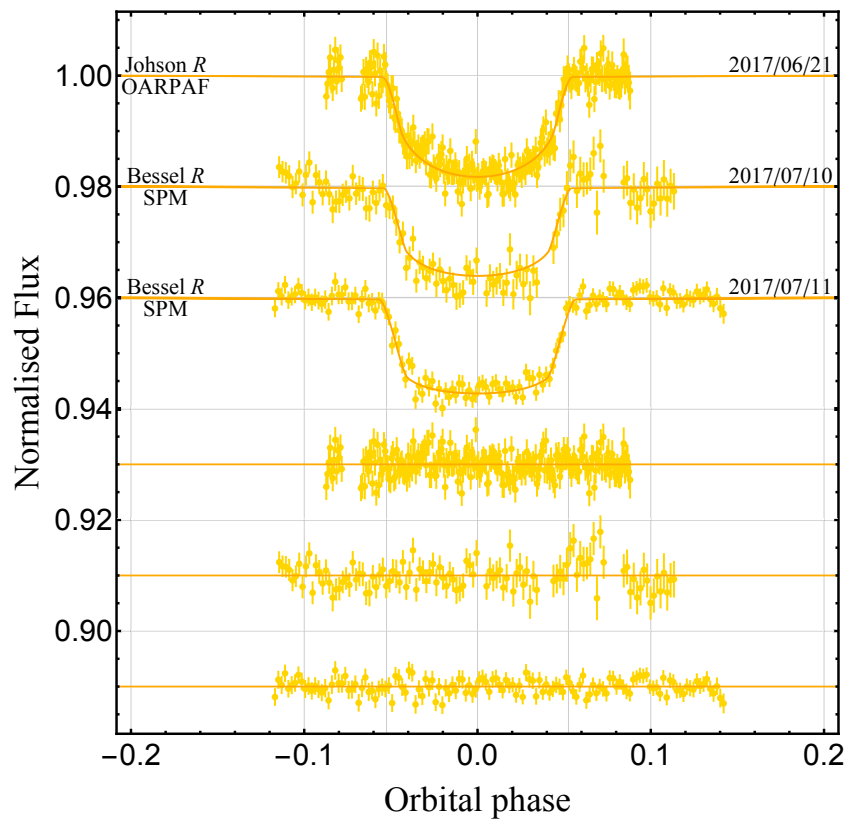

Figure 7. Light curves of two transits of KELT-16b observed with the SPM $84 \mathrm{~cm}$ telescope and one with the OARPAF $80 \mathrm{~cm}$ telescope. Observations were performed through two different $R$ filters. The labels indicate the observation date and the filter that was used for each observation. They are plotted versus the orbital phase and compared to the best-fitting JКTEВОР models. The residuals of the fits are plotted at the base of the figure.

Figs. 4, 5, 6, 7. The values of the photometric parameters, which resulted from the fit of each light curve, were combined into weighted means to get the final values. They are reported in Table 2.

\section{TRANSIT TIME ANALYSIS}

\subsection{Fitting the timing data}

From the study of our new light curves (see Sect. 4), we can estimate the mid-transit time for each of the transits that we recorded. However, we have to consider that time-correlated errors (i,e., red noise) can significantly affect ground-based data and, therefore, the determination of transit times. In order to derive more realistic uncertainties for each point in our light curves, we assessed the red noise by using the $\beta$ approach (e.g., Gillon et al. 2006; Winn et al. 2008; Gibson et al. 2008; Nikolov et al. 2012; Southworth et al. 2012a; Mancini et al. 2013a. Practically, we inflated the error bars further by multiplying the data weights by a factor $\beta \geq 1$, which is a measurement of how close the data noise is to the Poisson approximation. The $\beta$ factor is found by binning the data and determining the ratio between the size of the residuals versus what would be expected if the data followed the Poisson distribution. We evaluated the values of the $\beta$ factor for between two and ten data points for each light curve, and adopted the largest $\beta$ value.

We then estimated the mid-transit time for each of the transits that we recorded by running the Јктевор code another time. In particular, concerning the four transits that we simultaneously observed with two telescopes (see Appendix A), we obtained the following values for the mid-transit times:

\begin{tabular}{lcc}
\hline Telescope & \multicolumn{1}{c}{$T_{\text {mid }}\left(\mathrm{BJD}_{\mathrm{TDB}}\right)$} \\
\hline $\mathbf{2 0 1 7 . 0 6 . 2 1}$ & & \\
CA $1.23 \mathrm{~m}$ & $t_{1}=2457926.51170 \pm 0.00048$ \\
Cassini $1.52 \mathrm{~m}$ & $t_{2}=2457926.51199 \pm 0.00071$ \\
OARPAF $80 \mathrm{~cm}$ & $t_{3}=2457926.51419 \pm 0.00052$ \\
& $t_{2}-t_{1}=(25.1 \pm 102.8) \mathrm{sec}$ & $0.3-\sigma$ \\
& $t_{3}-t_{1}=(215.1 \pm 106.3) \mathrm{sec}$ & $3.5-\sigma$ \\
& $t_{3}-t_{2}=(190.1 \pm 86.4) \mathrm{sec}$ & $1.5-\sigma$
\end{tabular}

2017.06.22

CA $1.23 \mathrm{~m}$

Cassini $1.52 \mathrm{~m}$

$t_{1}=2457927.47825 \pm 0.00047$

$t_{2}=2457927.48150 \pm 0.00026$

$t_{2}-t_{1}=(280.8 \pm 63.1) \mathrm{sec} \quad 6.1-\sigma$

2018.07.02

CA $1.23 \mathrm{~m}$

Cassini $1.52 \mathrm{~m}$

$t_{1}=2458302.48199 \pm 0.00037$

$t_{2}=2458302.48202 \pm 0.00087$

$t_{2}-t_{1}=(2.6 \pm 107.1) \mathrm{sec} 0.03-\sigma$

2018.07.03

CA $1.23 \mathrm{~m}$

Cassini $1.52 \mathrm{~m}$

$t_{1}=2458303.44916 \pm 0.00093$

$t_{2}=2458303.44984 \pm 0.00091$

$t_{2}-t_{1}=(58.8 \pm 159.0) \mathrm{sec} \quad 0.5-\sigma$

We noticed that when the transit is simultaneously and completely monitored by two telescopes, including the out-of-transit phases, the difference between the mid-transit times is minimal $(\sim 2.6 \mathrm{sec}$, with $0.03-\sigma)$. Instead, when the transit is not completely monitored by the two telescopes, then the difference is greater $(\sim 215 \mathrm{sec}$ and $\sim 280 \mathrm{sec}$ in our worst cases, i.e. a difference of 3.5- $\sigma$ and 6.1$\sigma$, respectively). This happening was already noted by Barros et al. (2013). This stresses how important it is to get complete light curves (including the out-of-transit points) of transit events in order to achieve reliable estimates of the mid-transit times. Considering the differences in the above table, we decided to reject the timing from OARPAF $80 \mathrm{~cm}$ taken on 2017.06.21 and that from CA taken on 2017.06.22. For the remaining timings of the table, as in previous works of our series, we decide to take the weighted mean of the times for each of these four transits.

Finally, we assembled a final list of 69 transit timings by joining our measurements with those from Oberst et al. (2017), Maciejewski et al. (2018a) and Patra et al. (2020). They are reported in Table 3.

We selected the reference epoch as that corresponding to the first observation of our campaign (see Fig. 10) and we made several attempts to fit the above-mentioned list of timing data. In particular, we tried both a linear and a quadratic ephemeris in the forms

$T_{\text {mid }}=T_{0}+P_{\text {orb }} E$,

$T_{\text {mid }}=T_{0}+P_{\text {orb }} E+\frac{1}{2} \frac{d P_{\text {orb }}}{d E} E^{2}$.

As usual, $E$ represents the number of orbital cycles after the reference epoch $T_{0}$, while $\frac{d P_{\text {orb }}}{d E}$ is the change in the orbital period between succeeding transits. The fit of the mid-transit times with a straight line gave the following refinement of the linear transit ephemerides

$T_{\mathrm{mid}}=\mathrm{BJD}_{\mathrm{TDB}} 2457927.481100(80)+0.968993061(139) E$,

with a reduced chi-square of $\chi_{v}^{2}=0.98$ and a root-mean-square deviation (rmsd) scatter of $67.68 \mathrm{~s}$ (the quantities in brackets represent 


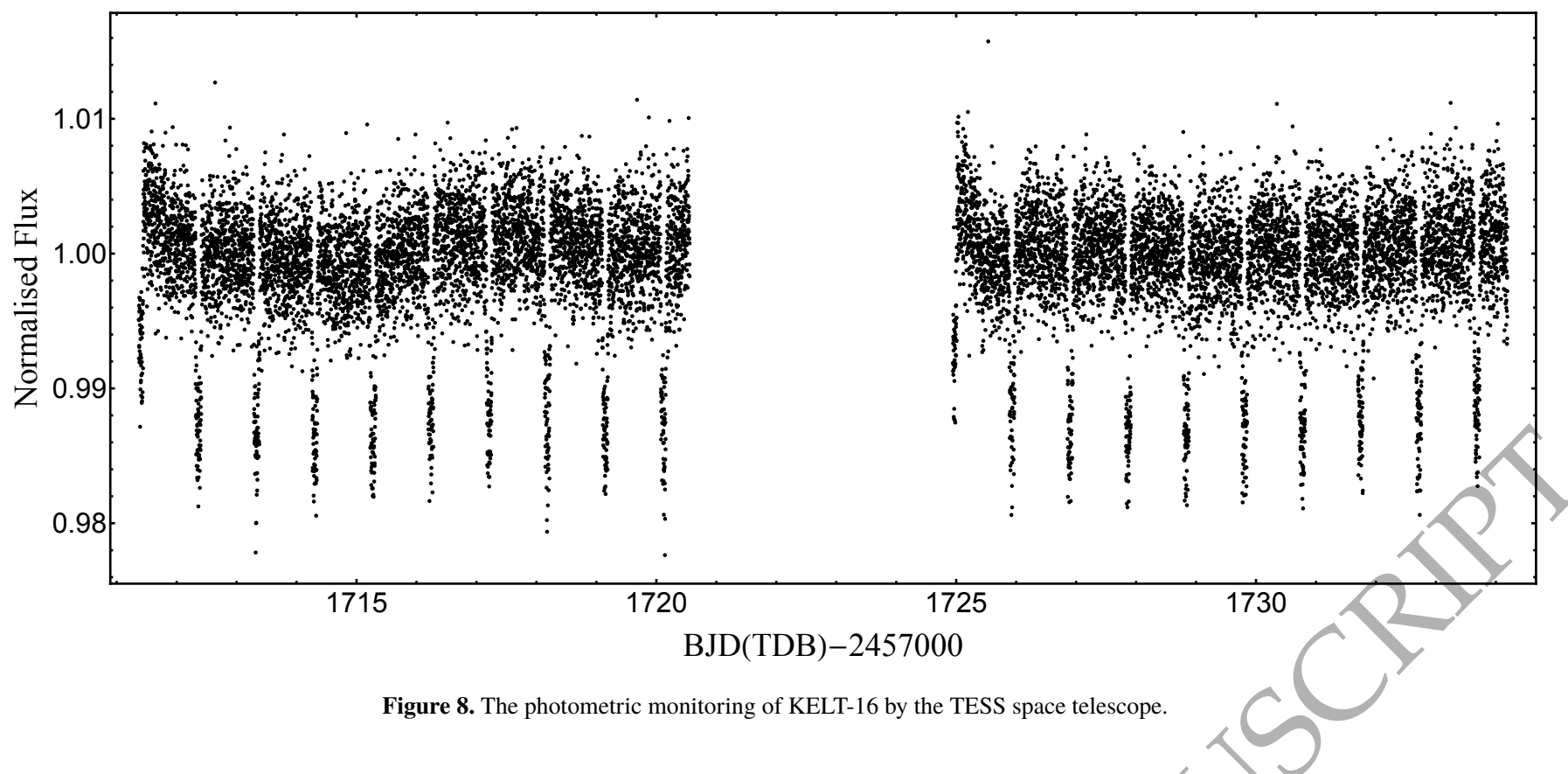

Table 2. Photometric properties of the KELT-16 system derived by fitting the transit light curves with JKTEBOP. The values reported in the third column are the weighted means of the results for the individual data sets and are compared with those from other works.

\begin{tabular}{llccc}
\hline Quantity & Symbol & This work & Oberst et al. (2017) & Maciejewski et al. (2018a) \\
\hline Sum of the fractional radii & $r_{\star}+r_{\mathrm{p}}$ & $0.33352 \pm 0.00237$ & - & - \\
Ratio of the fractional radii & $k$ & $0.10814 \pm 0.00087$ & $0.1070_{-0.0012}^{+0.0013}$ & $0.1076 \pm 0.0010$ \\
Orbital inclination......... & $i\left(^{\circ}\right)$ & $89.72 \pm 0.25$ & $84.4_{-2.3}^{+3.0}$ & $84.5_{-1.4}^{+2.0}$ \\
Star's fractional radius.... & $r_{\star}$ & $0.30131 \pm 0.00201$ & $0.310 \pm 0.012$ & $0.3088_{-0.0072}^{+0.000}$ \\
Planet's fractional radius.. & $r_{\mathrm{p}}$ & $0.03264 \pm 0.00032$ & - & - \\
\hline
\end{tabular}

the uncertainties in the preceding digits). Instead, the best-fitting quadratic ephemeris resulted to be

$$
\begin{array}{rl}
T_{\mathrm{mid}}=\mathrm{BJD}_{\mathrm{TDB}} 2457927 & 481114(82)+0.968993190(211) E+ \\
& -(1.62 \pm 2.01) \times 10^{-10} E^{2}
\end{array}
$$

with a slightly lower $\chi_{v}^{2}$ and rmsd scatter, i.e. 0.97 and $66.24 \mathrm{~s}$, respectively. We also tried a fit with a cubic ephemeris and, also in this case, we found a negligible improvement compared to the previous cases; see Table 4, where we summarise the results of our analysis.

Since the $\chi_{v}^{2}$ of the three models was very similar to each other, we estimate the Akaike Information Criterion (AIC) and the Bayesian Information Criterion (BIC). Both these criteria slightly prefer the linear model over the quadratic and cubic models (see Table 4). The timing residuals from the linear ephemeris are plotted in Fig. 10 together with the quadratic model.

Following a suggestion of the referee, we excluded the light curves that have missing ingress/egress or strong systematics and repeated the analysis of the transit times without these values to check if the results are consistent. In particular, we excluded the light curve recorded by the Cassini $1.52 \mathrm{~m}$ telescope on 2017/06/23 because the ingress is missing; we excluded the two T100 light curves (recorded on 2019/08/14 and 2019/09/16) because they were observed without using defocussing; we excluded the T100 light curve recorded on 2021/01/03 because the ingress is missing. Having excluded these four timings, we remade the TTV analysis and did not find significant differences compared to the case with the entire dataset.

\subsection{Orbital-decay analysis}

The analysis presented in the Sect. 5.1 shows that both linear and quadratic models fit the mid-transit times of KELT-16 b equally well. The impasse can only be overcome by acquiring new planetarytransit measurements. The quadratic terms can be progressively constrained to be smaller and smaller as more data are added. Based on the current data and following the approach of previous studies (e.g., Maciejewski et al. 2016; Patra et al. 2017; Southworth et al. $2019)$, we found that the change in the orbital period is $\frac{d P_{\text {orb }}}{d E}=$ $-(3.2 \pm 4.0) \times 10^{-10}$ days per orbital cycle and, therefore, the period derivative is $\dot{P}_{\text {orb }}=\frac{1}{P_{\text {orb }}} \frac{d P_{\text {orb }}}{d E}=-10.6 \pm 13.1 \mathrm{~ms} \mathrm{yr}^{-1}$, consistent with a constant orbital period or with an orbital period that shrink to zero in a time larger than $8 \mathrm{Myr}$.

The rate of the orbital decay, which we have deduced, may be used to limit the modified tidal dissipation quality factor, via Eq. (1), to $Q_{\star}^{\prime}>(1.9 \pm 0.8) \times 10^{5}$; this result is based on the $95 \%$ confidence lower limits on $\dot{P}_{\text {orb }}$, while the uncertainties come from propagating the errors in $M_{\mathrm{p}} / M_{\star}$ and $R_{\star} / a$ (see Table 5). 

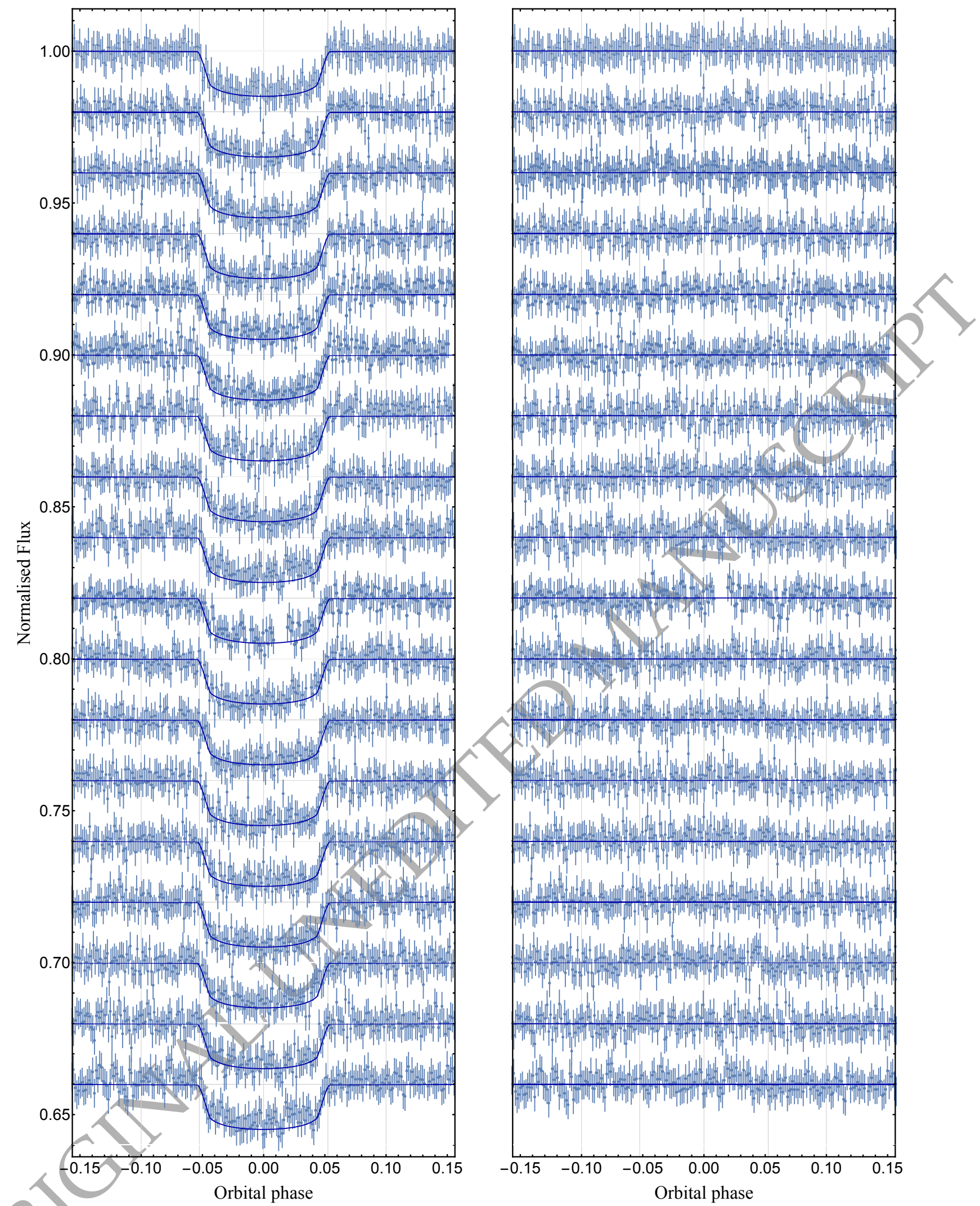

Figure 9. Left-hand panel: TESS light curves of KELT-16 b transits. They are plotted versus the orbital phase and compared to the best-fitting JKTEBOP models. Right-hand panel: the residuals of each fit. 


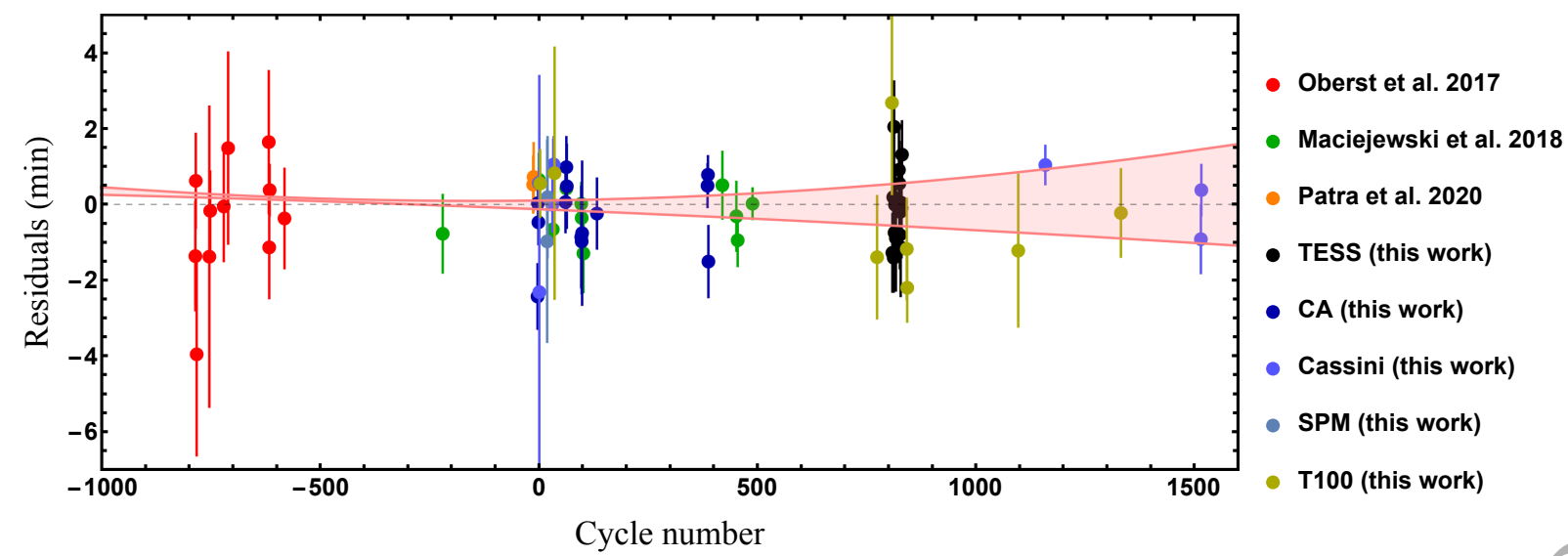

Figure 10. Plot of the residuals of the timings of mid-transit of KELT-16b versus a linear ephemeris. The points are coloured based on their source (see the legend). CA points (dark blue) include the times averaged on simultaneous observations (see Table 1 and Appendix A). The red band represents $1 \sigma$ uncertainty on the orbital decay model. Zooms in to the two best-sampled regions are shown in Fig. B1.

\section{PHYSICAL PROPERTIES}

\subsection{Analysis of stellar parameters}

We reviewed the stellar parameters of the KELT-16 star by fitting its Spectral Energy Distribution (SED) using the MESA Isochrones and Stellar Tracks (MIST; Dotter 2016; Choi et al. 2016) through the EXOFASTv2 suite (Eastman et al. 2019). To be precise, following Barbato et al. (2020), we fitted the stellar magnitudes (Oberst et al. 2017), imposing Gaussian priors on the effective temperature and the metallicity. These priors were based on the spectroscopic measurements and the parallax obtained from Gaia DR3. From this analysis, we estimated the stellar atmospheric parameters, which resulted to be in good agreement with those of the discovery paper, see Table 5.

\subsection{Physical parameters of the planetary system}

The main physical properties of the KELT-16 system were determined using our robust "Homogeneous Studies" approach (see for example Southworth 2012; Mancini et al. 2013a and references therein), which makes use of the photometric parameters reported in Table 2, the stellar radial-velocity amplitude $K \star \star 494 \pm 25 \mathrm{~m} \mathrm{~s}^{-1}$ (Oberst et al. 2017), the spectroscopic parameters (Sect. 6.1) and a set of theoretical stellar models. We calculated the properties of the system by using standard formulae, including the velocity amplitude of the planet, $K_{\mathrm{p}}$. Then, we iteratively adjusted the value of $K_{\mathrm{p}}$ to find the best agreement between the values of $r_{\star}$ and $T_{\text {eff }}$ that we measured from the observations and those predicted by a single theoretical model of the calculated mass. This procedure was repeated for a wide grid of ages and five different theoretical models.

Finally, we took the unweighted mean of the five sets of values as the final set of physical properties of the system. They are reported in Table 5. The systematic uncertainties of these values were calculated as the standard deviation of the results from the five models for each output parameter. Table 5 also shows the physical properties found by other authors. Our results are in good agreement with them but more precise because of the much more extensive photometry of the system presented in the current work.

\subsection{Variation of the planetary radius with the wavelength}

It has been well ascertained that the transmission spectra of hot Jupiters show characteristic absorption features at particular wavelengths (see, e.g., Sing 2018). In particular, those for which the incident stellar flux is $>10^{9} \mathrm{erg} \mathrm{s}^{-1} \mathrm{~cm}^{-2}$ are expected to host a large amount of absorbing substances in their atmospheres, such as gaseous titanium oxide (TiO) and vanadium oxide (VO), acting in a window of the optical region between 450 and $700 \mathrm{~nm}$ (Fortney et al. 2008). Using the multi-colour light curves that we took through different passbands (i.e. $B, V, R^{7}, I$ ), we attempted to reconstruct a low-resolution transmission spectrum of KELT-16b. We excluded from this analysis the low-quality and the incomplete light curves that we presented in Sect. 3 (also see Table 1 and Figs 4, 5 and 7), because from their fits we can get inaccurate value of the transit depth. We also excluded the TESS light curves because the long-pass filter of TESS is just too wide $(>500 \mathrm{~nm})$ for our purposes.

Following a general approach, we run again Јктевор for each of our light curves to calculate the ratio of the radii in each passband, fixing this time the other photometric parameters to their best-fitting values that we previously estimated (see Table 5). This returned a set of $k=R_{\mathrm{p}} / R_{\star}$ values which are directly comparable and whose error bars exclude common sources of uncertainty. Then, we made a weighted mean of these values for each of the four passbands, obtaining the following: $k_{B}=0.10767 \pm$ $0.00055, k_{V}=0.10846 \pm 0.00076, k_{R}=0.10825 \pm 0.00112$ and $k_{I}=0.10833 \pm 0.00051$. These values are shown in Fig 11 and compared with two one-dimensional model atmospheres, which were obtained by Fortney et al. (2010). In particular, the red line has been calculated for Jupiter's gravity $\left(25 \mathrm{~m} \mathrm{~s}^{-2}\right)$ with a base radius of $1.25 R_{\mathrm{Jup}}$ at the $10 \mathrm{bar}$ level and at $2500 \mathrm{~K}$. The opacity of TiO and VO molecules is excluded from the model and the optical transmission spectrum is dominated by $\mathrm{H}_{2} / \mathrm{He}$ Rayleigh scattering in the blue, and pressure-broadened neutral atomic lines of sodium at $589 \mathrm{~nm}$ and potassium at $770 \mathrm{~nm}$. The green line represents a model similar to the previous one, but the opacity of $\mathrm{TiO}$ and $\mathrm{VO}$ molecules was included. This model shows significant optical absorption that broadly peaks around $700 \mathrm{~nm}$, with a sharp fall-off in the blue and a

${ }^{7}$ Even though different $R$ filters were used, they are very similar considering their bandwidth and effective wavelength. 
Table 3. Times of mid-transit for KELT- $16 \mathrm{~b}$ and their residuals for a constant period.

\begin{tabular}{|c|c|c|c|}
\hline $\begin{array}{l}\text { Time of minimum } \\
\text { BJD(TDB) }-2400000\end{array}$ & $\begin{array}{r}\text { Cycle } \\
\text { no. }\end{array}$ & $\begin{array}{r}\mathrm{O}-\mathrm{C} \\
\text { (day) }\end{array}$ & Reference \\
\hline $57165.85142 \pm 0.00099$ & -786.0 & -0.00095 & Oberst et al. (2017) \\
\hline $57166.82179 \pm 0.00086$ & -785.0 & 0.00043 & Oberst et al. (2017) \\
\hline $57168.75660 \pm 0.00185$ & -783.0 & -0.00275 & Oberst et al. (2017) \\
\hline $57196.85920 \pm 0.00275$ & -754.0 & -0.00096 & Oberst et al. (2017) \\
\hline $57198.79802 \pm 0.00072$ & -752.0 & -0.00012 & Oberst et al. (2017) \\
\hline $57228.83690 \pm 0.00100$ & -721.0 & -0.00004 & Oberst et al. (2017) \\
\hline $57238.52790 \pm 0.00175$ & -711.0 & 0.00103 & Oberst et al. (2017) \\
\hline $57328.64440 \pm 0.00130$ & -618.0 & 0.00114 & Oberst et al. (2017) \\
\hline $57329.61146 \pm 0.00093$ & -617.0 & -0.00079 & Oberst et al. (2017) \\
\hline $57330.58151 \pm 0.00046$ & -616.0 & 0.00026 & Oberst et al. (2017) \\
\hline $57363.52676 \pm 0.00091$ & -582.0 & -0.00026 & Oberst et al. (2017) \\
\hline $57714.30206 \pm 0.00071$ & -220.0 & -0.00054 & Maciejewski et al. (2018a) \\
\hline $57914.88456 \pm 0.00051$ & -13.0 & 0.00036 & Patra et al. (2020) \\
\hline $57915.85370 \pm 0.00062$ & -12.0 & 0.00050 & Patra et al. (2020) \\
\hline $57924.57245 \pm 0.00059$ & -3.0 & -0.00169 & $\mathrm{CA}$ \\
\hline $57925.54315 \pm 0.00044$ & -2.0 & 0.00002 & $\mathrm{CA}$ \\
\hline $57926.51179 \pm 0.00040$ & -1.0 & -0.00033 & $\mathrm{CA} /$ Cassini \\
\hline $57927.48150 \pm 0.00026$ & 0.0 & 0.00039 & Cassini \\
\hline $57927.48156 \pm 0.00047$ & 0.0 & 0.00045 & Maciejewski et al. (2018a) \\
\hline $57928.44850 \pm 0.00396$ & 1.0 & -0.00161 & Cassini \\
\hline $57930.38847 \pm 0.00061$ & 3.0 & 0.00038 & $\mathrm{~T} 100$ \\
\hline $57945.89131 \pm 0.00184$ & 19.0 & -0.00068 & SPM \\
\hline $57946.86111 \pm 0.00110$ & 20.0 & 0.00013 & SPM \\
\hline $57957.51989 \pm 0.00049$ & 31.0 & -0.00001 & Cassini \\
\hline $57958.48844 \pm 0.00026$ & 32.0 & -0.00046 & Maciejewski et al. (2018a) \\
\hline $57959.45852 \pm 0.00055$ & 33.0 & 0.00063 & Cassini \\
\hline $57960.42762 \pm 0.00050$ & 34.0 & 0.00073 & Cassini \\
\hline $57962.36544 \pm 0.00230$ & 36.0 & 0.00057 & $\mathrm{~T} 100$ \\
\hline $57986.58974 \pm 0.00055$ & 61.0 & 0.00004 & $\mathrm{CA}$ \\
\hline $57988.52836 \pm 0.00055$ & 63.0 & 0.00068 & $\mathrm{CA}$ \\
\hline $57988.52797 \pm 0.00038$ & 63.0 & 0.00029 & Maciejewski et al. (2018a) \\
\hline $57989.49700 \pm 0.00076$ & 64.0 & 0.00033 & $\mathrm{CA}$ \\
\hline $58021.47285 \pm 0.00092$ & 97.0 & -0.00060 & $\mathrm{CA}$ \\
\hline $58021.47346 \pm 0.00038$ & 97.0 & 0.00001 & Maciejewski et al. (2018a) \\
\hline $58022.44176 \pm 0.00095$ & 98.0 & -0.00068 & $\mathrm{CA}$ \\
\hline $58022.44219 \pm 0.00046$ & 98.0 & -0.00025 & Maciejewski et al. (2018a) \\
\hline $58023.41090 \pm 0.00131$ & 99.0 & -0.00053 & $\mathrm{CA}$ \\
\hline $58026.31752 \pm 0.00071$ & 102.0 & -0.00090 & Maciejewski et al. (2018a) \\
\hline $58056.35704 \pm 0.00064$ & 133.0 & -0.00017 & $\mathrm{CA}$ \\
\hline $58301.51280 \pm 0.00039$ & 386.0 & 0.00034 & $\mathrm{CA}$ \\
\hline $58302.48200 \pm 0.00034$ & 387.0 & 0.00054 & $\mathrm{CA} /$ Cassini \\
\hline $58303.44940 \pm 0.00065$ & 388.0 & -0.00105 & $\mathrm{CA} /$ Cassini \\
\hline $58334.45858 \pm 0.00061$ & 420.0 & 0.00035 & Maciejewski et al.(2018a) \\
\hline $58365.46578 \pm 0.00063$ & 452.0 & -0.00022 & Maciejewski etal (2018a) \\
\hline $58368.37232 \pm 0.00047$ & 455.0 & -0.00066 & Maciejewski et al. (2018a) \\
\hline $58401.31876 \pm 0.00028$ & 489.0 & 0.00001 & Maciejewski et al. (2018a) \\
\hline $58677.48078 \pm 0.00112$ & 774.0 & -0.00097 & $\mathrm{~T} 100$ \\
\hline $58710.42937 \pm 0.00167$ & 808.0 & 0.00186 & \\
\hline $58711.39561 \pm 0.00071$ & 809.0 & -0.00089 & TESS \\
\hline $58713.33461 \pm 0.00049$ & 811.0 & 0.00013 & TESS \\
\hline $58714.30250 \pm 0.00062$ & 812.0 & -0.00098 & TESS \\
\hline $58715.27389 \pm 0.00083$ & 813.0 & 0.00142 & TESS \\
\hline $58716.24094 \pm 0.00060$ & 814.0 & -0.00052 & TESS \\
\hline $58717.21045 \pm 0.00053$ & 815.0 & -0.00001 & TESS \\
\hline $58719.14782 \pm 0.00096$ & 817.0 & -0.00062 & TESS \\
\hline $58725.93202 \pm 0.00074$ & 824.0 & 0.00063 & TESS \\
\hline $58726.90076 \pm 0.00076$ & & 0.00038 & TESS \\
\hline $58727.86882 \pm 0.00061$ & 826.0 & -0.00056 & TESS \\
\hline $58728.83823 \pm 0.00054$ & 827.0 & -0.00014 & TESS \\
\hline $58729.80658 \pm 0,00089$ & 828.0 & -0.00079 & TESS \\
\hline $58730.77638 \pm 0.00067$ & 829.0 & 0.00003 & TESS \\
\hline $58731.74528 \pm 0.00090$ & 830.0 & -0.00007 & TESS \\
\hline $58732.71525 \pm 0.00061$ & 831.0 & 0.00091 & TESS \\
\hline $58733.68336 \pm 0.00077$ & 832.0 & 0.00003 & TESS \\
\hline $58743.37244 \pm 0.00092$ & 842.0 & -0.00082 & $\mathrm{~T} 100$ \\
\hline $58744.34073 \pm 0.00062$ & 843.0 & -0.00153 & $\mathrm{~T} 100$ \\
\hline $58990.46559 \pm 0.00139$ & 1097.0 & -0.00085 & $\mathrm{~T} 100$ \\
\hline $59050.54472 \pm 0.00035$ & 1159.0 & 0.00072 & Cassini \\
\hline $59218.17959 \pm 0.00080$ & 1332.0 & -0.00016 & $\mathrm{~T} 100$ \\
\hline $59395.50478 \pm 0.00062$ & 1515.0 & -0.00064 & Cassini \\
\hline $59396.47467 \pm 0.00046$ & 1516.0 & 0.00026 & Cassini \\
\hline
\end{tabular}

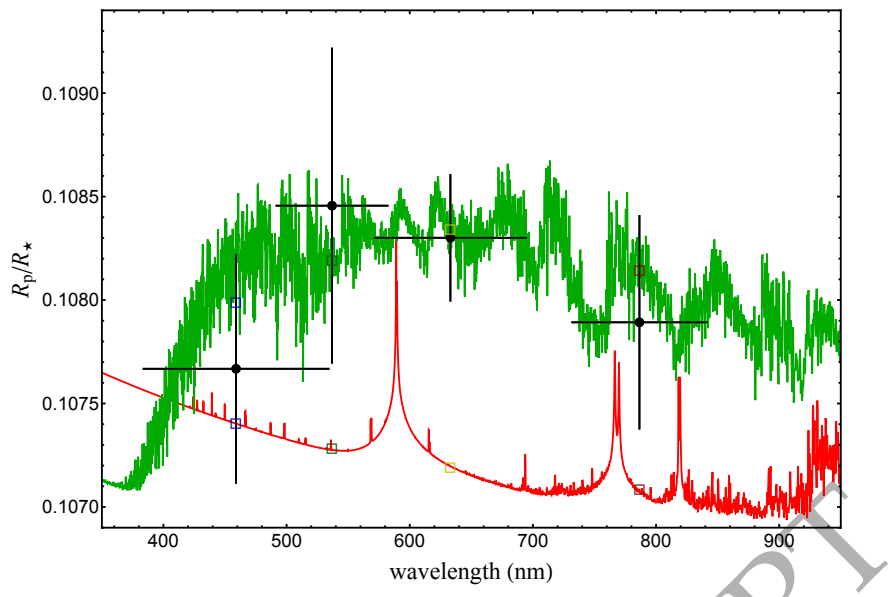

Figure 11. Variation of the planetary radius, in terms of the planet/star radius ratio, with wavelength. The points are from the ground-based transit observations presented in this work. The vertical bars represent the errors in the measurements and the horizontal bars show the FWHM transmission of the passbands used. The observational points are compared with two synthetic spectra from Fortney et al. (2010) (see text for details). Coloured squares represent band-averaged model radii over the bandpasses of the observations.

shallower fall-off in the red. Unfortunately, the accuracy of our data is not at the same level of accuracy as the atmosphere models; even though they indicate a possible radius variation between wavelength ranges $350-420 \mathrm{~nm}$ and $500-800 \mathrm{~nm}$, practically, they show a flat transmission spectrum to within the experimental uncertainties.

\subsection{Flux ratio of KELT-16 $b$ from TESS phase curve}

Due to its short period, KELT-16b has been selected as a backup target for the JWST Early Release Science program (Bean et al. 2018). This because the time required to observe an its full orbit phase curve is relatively small when compared with a more typical hot Jupiter $\left(P_{\text {orb }} \sim 3\right.$ days). Moreover, KELT-16 b is highly irradiated and, therefore, will give a large thermal emission signal.

By using the SPOC pipeline, we extracted the data of KELT16 from the TESS database and generated a phase curve with the periodicity and time of transit shown in Table 5. We exploited the Starry python package (Luger et al. 2019) to fit both the transit and the secondary eclipse to measure the flux ratio of the planet over its star, see Fig 12. We approximate KELT-16 b to be tidally-locked and model it with a simple dipole brightness map, where the bright side is facing the star, but we also take into account a possible offset that captures the eventual misalignment between the hot spot of the planet and the sub-stellar point, which causes the maximum of the planet flux to happen outside of the secondary eclipse. The mass and radius of both the star and the planet are set to be normally distributed around the value and standard deviation from Table 5, while the offset and the amplitude of the planet flux are free to vary within reasonable limits. A brightness temperature for both the day side and the night side of the planet is then computed with the Planck's law. The results are shown in Table 6 and the distributions are plotted in Fig. C1, from where result an offset of $25 \pm 14^{\circ}$ and a $F_{\mathrm{p}} / F_{\star}$ of $434 \pm 42 \mathrm{ppm}$. Finally, the brightness temperature of the day- and night-side simple model are $T_{\text {day }}=3190 \pm 61 \mathrm{~K}$ and $T_{\text {night }}=2668 \pm 56 \mathrm{~K}$, respectively. These values are compatible within $1 \sigma$ and $2 \sigma$, respectively, of those found by Bell et al. (2021) with Spitzer measurements. 
Table 4. Summary of the best-fitting values of the parameters obtained by using a linear-, a quadratic and a cubic-ephemeris model for the KELT-16 $\mathrm{b}$ time residuals. The corresponding reduced chi-square, AIC, BIC and root-mean-square-deviation (rmsd) values are also reported.

\begin{tabular}{|c|c|c|c|c|c|c|c|c|}
\hline Poly order & $\chi_{v}^{2}$ & $T_{0}\left(\mathrm{BJD}_{\mathrm{TDB}}-2400000\right)$ & Orbital Period (day) & Quadratic term & Cubic term & AIC & $\mathrm{BIC}$ & $\operatorname{rmsd}(\mathrm{s})$ \\
\hline Linear & 0.98 & $57927.481100 \pm 0.000080$ & $0.968993061 \pm 0.000000139$ & - & - & 73.29 & 77.82 & 67.68 \\
\hline Quadratic & 0.97 & $57927.481114 \pm 0.000082$ & $0.968993190 \pm 0.000000211$ & $-(1.62 \pm 2.01) \times 10^{-10}$ & - & 74.64 & 81.43 & 66.49 \\
\hline Cubic & 0.96 & $57927.481145 \pm 0.000095$ & $0.968993123 \pm 0.000000236$ & $-(3.49 \pm 3.54) \times 10^{-10}$ & $(1.81 \pm 2.82) \times 10^{-13}$ & 76.23 & 85.28 & 65.97 \\
\hline
\end{tabular}

Table 5. Physical parameters of the planetary system KELT-16 derived in this work (Sect. 6.2), compared with those from other works. Where two error bars are given, the first refers to the statistical uncertainties, while the second to the systematic errors. Notes. ${ }^{a}$ This value was obtained from the SED fitting procedure (Sect. 6.1). ${ }^{b}$ This value was obtained from the transit-time analysis (Sect. 5).

\begin{tabular}{|c|c|c|c|c|c|c|}
\hline Quantity & Symbol & Unit & This work & Oberst et al. (2017) & Maciejewski et al. (2018a) & Patra et al. (2020) \\
\hline \multicolumn{7}{|l|}{ Stellar parameters } \\
\hline Stellar effective temperature ${ }^{a}$ & $T_{\text {eff }}$ & $\mathrm{K}$ & $6237_{-53}^{+55}$ & $6236 \pm 54$ & - & \\
\hline Stellar metallicity ${ }^{a}$ & {$[\mathrm{Fe} / \mathrm{H}]$} & dex & $-0.006_{-0.082}^{+0.082}$ & $-0.002_{-0.085}^{+0.086}$ & - & \\
\hline Stellar mass ..... & $M_{\star}$ & $M_{\odot}$ & $1.195 \pm 0.037 \pm 0.024$ & $1.211_{-0.046}^{+0.043}$ & - & \\
\hline Stellar radius $\ldots \ldots \ldots \ldots \ldots$ & $R_{\star}$ & $R_{\odot}$ & $1.315 \pm 0.016 \pm 0.009$ & $1.360_{-0.053}^{+0.064}$ & - & \\
\hline Stellar surface gravity ....... & $\log g_{\star}$ & cgs & $4.278 \pm 0.007 \pm 0.003$ & $4.253_{-0.036}^{+0.031}$ & - & - \\
\hline Stellar density . ........... & $\rho_{\star}$ & $\rho_{\odot}$ & $0.5256 \pm 0.0100$ & $0.481_{-0.057}^{+0.056}$ & & - \\
\hline Age $\ldots \ldots \ldots \ldots \ldots \ldots$ & $\tau$ & Gyr & $3.0_{-0.5-0.4}^{+0.7+0.4}$ & $3.1 \pm 0.3$ & & - \\
\hline$V$-band extinction ${ }^{a} \ldots \ldots \ldots$ & $A_{V}$ & mag & $0.179_{-0.054}^{+0.055}$ & $0.04 \pm 0.04$ & & - \\
\hline Parallax $^{a}$ & $\varpi$ & mas & $2.247_{-0.013}^{+0.013}$ & - & & - \\
\hline Distance $^{a}$ & $d$ & $\mathrm{pc}$ & $445.0 \pm 2.6$ & $399 \pm 19$ & & - \\
\hline \multicolumn{7}{|l|}{ Planetary parameters } \\
\hline Planetary mass . ............ & $M_{\mathrm{p}}$ & $M_{\text {Jup }}$ & $2.71 \pm 0.15 \pm 0.04$ & $2.75_{-0.15}^{+0.16}$ & - & - \\
\hline Planetary radius $\ldots \ldots \ldots \ldots$ & $R_{\mathrm{p}}$ & $R_{\text {Jup }}$ & $1.383 \pm 0.023 \pm 0.009$ & $1.415_{-0.067}^{+0.084}$ & - & - \\
\hline Planetary surface gravity .... & $g_{\mathrm{p}}$ & $\mathrm{m} \mathrm{s}^{-2}$ & $35.1 \pm 2.0$ & $33.9_{-3.6}^{+3.401}$ & - & - \\
\hline Planetary density $\ldots \ldots \ldots \ldots$ & $\rho_{\mathrm{p}}$ & $\rho_{\text {Jup }}$ & $0.958 \pm 0.062 \pm 0.006$ & $0.90 \pm 0.14$ & - & - \\
\hline Equilibrium temperature .... & $T_{\mathrm{eq}}$ & $\mathrm{K}$ & $2417 \pm 22$ & & - & - \\
\hline Safronov number . .......... & $\Theta$ & & $0.0667 \pm 0.0036 \pm 0.0004$ & $4 \pm 0.0045$ & - & - \\
\hline \multicolumn{7}{|l|}{ Orbital parameters } \\
\hline Semi-major axis $\ldots \ldots \ldots \ldots$ & $a$ & au & $0.02035 \pm 0.0002$ & $0.02044_{-0.00026}^{+0.00024}$ & - & - \\
\hline Inclination $\ldots \ldots \ldots \ldots \ldots$ & $i$ & degree & & $84.4_{-2.3}^{+3.0}$ & $84.5_{-1.4}^{+2.0}$ & - \\
\hline Constant period & & & & & & \\
\hline Time of mid-transit ${ }^{b}$ & $T_{0}$ & $\mathrm{BJD}_{\mathrm{TDB}}$ & $2457927.481100(80)$ & $2457165.85179(49)$ & $2457247.24774(24)$ & $2457910.03913(11)$ \\
\hline $\begin{array}{l}\text { Period }^{b} \ldots \ldots \ldots \ldots \ldots \ldots \\
\quad \text { Orbital decay }\end{array}$ & $P_{\text {orb }}$ & days & $0.96899306(14)$ & $0.9689951(24)$ & $0.96899320(29)$ & $0.96899319(30)$ \\
\hline Time of mid-transit ${ }^{b}$ & $T_{0}$ & $\mathrm{BJD}_{\mathrm{TDB}}$ & $14(82)$ & - & - & $2457910.03918(15)$ \\
\hline Period $^{b} \ldots \ldots \ldots \ldots \ldots$ & $P_{\text {orb }}$ & days & $0.96899319(21)$ & - & - & $0.96899314(33)$ \\
\hline
\end{tabular}

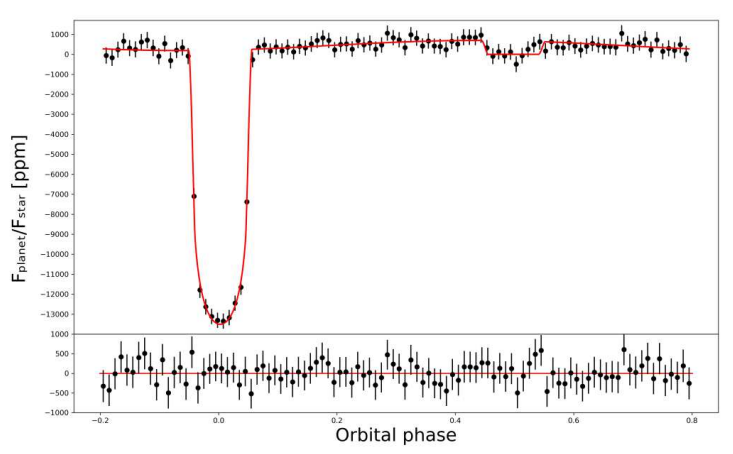

Figure 12. Top panel: phase curve of KELT-16 from TESS data, fitted with the Starry package. Bottom panel: residual of the fit.
Table 6. Posterior values and their standard deviations over the model parameters from a Starry computation with 8000 draw iterations. The day and the night flux are defined as the fluxes ratio that the planet emits over the star's respectively at phase 0.5 and 0.0 in ppm (also the average planet amplitude is in ppm). hdi_3\% and hdi_97\% represent the lower and upper bounds of a $\sim 95 \%$ credible interval, which contains the true parameter value with $\sim 95 \%$ probability.

\begin{tabular}{lccccc}
\hline Quantity & Unit & This work & hdi_3\% & hdi_97\% & Bell et al. (2021) \\
\hline$F_{\mathrm{p}} / F_{\star} \ldots \ldots$ & $(\mathrm{ppm})$ & $434.19 \pm 41.61$ & 361.08 & 519.88 & $4810_{-310}^{+330}$ \\
Max flux offset & ${ }^{\circ} \mathrm{E}$ & $25.25 \pm 14.03$ & -0.56 & 52.50 & $-38_{-15}^{+16}$ \\
Day flux $\ldots \ldots$ & $(\mathrm{ppm})$ & $654.61 \pm 71.18$ & 521.35 & 786.51 & - \\
Night flux $\ldots .$. & $(\mathrm{ppm})$ & $213.78 \pm 31.94$ & 158.69 & 276.11 & - \\
$T_{\text {day }} \ldots \ldots \ldots \ldots$ & $\mathrm{K}$ & $3190 \pm 61$ & 3077 & 3301 & $3070_{-150}^{+160}$ \\
$T_{\text {night }} \ldots \ldots \ldots$ & $\mathrm{K}$ & $2668 \pm 56$ & 2563 & 2773 & $1900_{-440}^{+430}$ \\
\hline
\end{tabular}



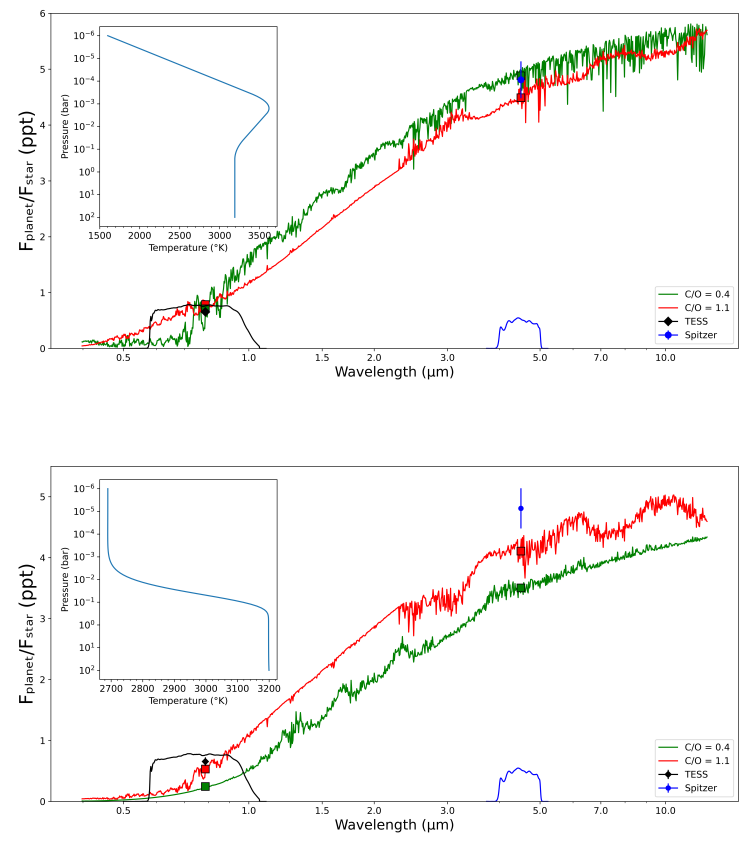

Figure 13. Dayside emission spectra of KELT-16b in terms of the planetto-star flux ratio, with a specimen atmosphere rich in hydrogen and with two different $\mathrm{C} / \mathrm{O}$ ratios and temperature profiles. The spectra in green and red correspond to $\mathrm{C} / \mathrm{O}=0.4$ and $\mathrm{C} / \mathrm{O}=1.1$ atmospheres, respectively, with a simple mixture of $\mathrm{H}_{2} \mathrm{O}, \mathrm{CO}, \mathrm{CH}_{4}, \mathrm{CO}_{2}, \mathrm{C}_{2} \mathrm{H}_{2}, \mathrm{HCN}$ and TiO. The temperature profiles are shown in the upper left inner panels, the top one being a temperature inversion model with a peak temperature at $3600 \mathrm{~K}$, while the bottom one is the Guillot (2010) model. The black diamond is the expected day flux ratio contribution from this paper with TESS data, while the blue dot is the calculated planet flux ratio with Spitzer from Bell et al. (2021). The bandpass integrated model points are shown in the coloured squares. The two curves at the bottom of the panels show the TESS and the Spitzer $4.5 \mu \mathrm{m}$ photometric bandpasses.

\subsection{Emission spectrum of KELT-16 b}

In order to compare the calculated flux ratio at the TESS wavelength (centred on $786.5 \mathrm{~nm}$ ) with that at the Spitzer wavelength (centered on $4500 \mathrm{~nm}$ ) from Bell et al. (2021), we used the Taurex python package (Al-Refaie et al. 2019) and generated four emission-spectrum models (Fig. 13), which are based on the KELT16 planetary-system parameters (Table 5), with two different atmosphere compositions ${ }^{8}$ (oxygen-dominated, $\mathrm{C} / \mathrm{O}=0.4$ in green; carbon-dominated, $\mathrm{C} / \mathrm{O}=1.1 \mathrm{in}$ red) and two different temperature profiles: a temperature inversion model for the top panel and the Guillot (2010) model for the bottom panel. In every emission model, we set a constant quench temperature $(T=3190 \mathrm{~K})$, i.e. the temperature of the lower atmosphere $(P \sim 1 \mathrm{bar})$, as it fits very well the planet flux ratio calculated with TESS data. However, from an inspection of the two panels, only the inversion-temperature case manages to also fit the flux ratio from Spitzer with a peak temperature of $3600 \mathrm{~K}$.

These atmosphere compositions are the same used by Madhusudhan et al. (2012) for WASP-19b, which has an equilibrium temperature and radius similar to those of KELT-16 b.

\section{SUMMARY AND CONCLUSIONS}

In this work we studied the physical and orbital properties of the ultra-hot Jupiter KELT-16 b, which is one of the few giant transiting exoplanets with $P_{\text {orb }}<1$ day and belongs to a group of exoplanets that are the most favourable to detect orbital decay (see discussion in Sect. 1.1 and Sect. 1.2). We reported the photometric monitoring of 28 transit events of KELT-16 b, which were observed with five medium-class telescopes through four different optical passbands. Most of the transits were observed using the defocussing technique, achieving a photometric precision of 1 mmag per observation in the best case (this unusual lack of good accuracy can be explained by the fact that there are no good comparison stars around KELT-16). Three transits were simultaneously observed with two different telescopes in different countries and one with three telescopes. In total, we collected 34 new light curves that were modelled with the јктевор code (see Figs. 4, 5, 6, 7). We also considered the TESS data and analysed 18 complete transits recorded by this space telescope (see Fig. 8). Our principal results are as follows.

- We estimated the mid-transit time for each of the transit event of KELT-16b that we presented. These timings were joined to others already published, obtaining a final list of 69 epochs (Table 3), which we used for updating the ephemeris of the orbital period and the mid-transit time. We also searched for evidence of a decrease in its orbital period. Our analysis shows the possibility of TTVs in this planetary system, the values of the $\chi_{v}^{2}$, AIB and BIC of the orbital-decay ephemeris model being similar to the constant orbital-period model. Longer monitoring of KELT-16 b transits are needed to obtain robust indications that the orbit of KELT-16b is decaying. Based on the current data and assuming that the period is not changing, we can set a limit of $Q_{\star}^{\prime}>(2.2 \pm 0.4) \times 10^{5}$ with $95 \%$ confidence.

- We have used the TESS and the new ground-based light curves to refine the physical parameters of the KELT-16 planetary system. Our results are shown in Table 5 and, in general, are in good agreement with those obtained by Oberst et al. (2017) but more precise.

- As stressed by Oberst et al. (2017), its ultra-short period and the high irradiation make KELT-16 $b$ a benchmark target for atmospheric studies. Taking advantage of our multi-band photometric observations, we reconstructed a low-resolution optical transmission spectrum of the planet. We found a small variation of the planet's radius, which suggests the presence of strong absorbers in the optical, as expected, but at a low significance. More precise observations are mandatory to robustly confirm this indication.

- Using the TESS data, we reconstructed the phase curve of the KELT-16 adopting the periodicity and the time of transit shown in Table 5. We simultaneously fitted the transit and occultation and estimated the flux ratio of the planet over its parent star and estimated the temperature of both the day- and night-side of the planet $(3190 \pm 61 \mathrm{~K}$ and $2668 \pm 56 \mathrm{~K}$, respectively). Moreover, we found that KELT- $16 \mathrm{~b}$ has a phase offset of $25 \pm 14^{\circ} \mathrm{E}$. These results (see Table 6), which are based on TESS data, are compatible with those found with Spitzer data (Bell et al. 2021).

- We compared the flux ratio at the TESS wavelength with that at the Spitzer wavelength and generated several emission-spectrum models for probing the chemical composition of the planet's atmo- 
sphere. We found that an atmosphere with temperature inversion is favoured, with a slight preference for an oxygen-dominated rather than a carbon-dominated composition. Again, many more measurements at different wavelength are needed to confirm this results.

\section{ACKNOWLEDGEMENTS}

This paper is based on observations collected with $(i)$ the Zeiss $1.23 \mathrm{~m}$ telescope at the Centro Astronómico Hispano Alemán (CAHA) at Calar Alto, Spain; (ii) the Cassini $1.52 \mathrm{~m}$ telescope, run by INAF-Osservatorio Astrofisico e Scienza dello Spazio di Bologna at Loiano, Italy; (iii) the T100 $1.0 \mathrm{~m}$ telescope at the TUG observatory, Turkey; (iv) the SPM $84 \mathrm{~cm}$ telescope at the National Astronomical observatory, Mexico; $(v)$ the $80 \mathrm{~cm}$ telescope at the OARPAF observatory, Italy. Operations at the Calar Alto telescopes are jointly performed by Junta de Andalucía and the Instituto de Astrofísica de Andalucía (CSIC) in Granada, Spain. We thank Roberto Gualandi for his technical assistance at the Cassini telescope. We thank the support astronomers of CAHA for their technical assistance at the Zeiss telescope. GD acknowledges support from CONICYT project Basal AFB-170002. OB acknowledges the support by The Scientific and Technological Research Council of Turkey (TÜBİTAK) with the project 118F042. We thank TÜBİTAK for the partial support in using T100 telescope with the project numbers 19AT100-1471 and 18BT100-1334. We thank the anonymous referee for their useful criticisms and suggestions that helped to improve the quality of this work. The following internet-based resources were used in research for this paper: the ESO Digitized Sky Survey; the NASA Astrophysics Data System; the SIMBAD database operated at CDS, Strasbourg, France; the arXiv scientific paper preprint service operated by the Cornell University.

\section{DATA AVAILABILITY}

The reduced light curves presented in this work will be made available at the CDS (http://cdsweb.u-strasbg.fr/).

\section{REFERENCES}

Adams E. R., López-Morales M., Elliot J. L., Seager S., Osip D. J., 2010, ApJ, 721, 1829

Al-Refaie A. F., Changeat Q., Waldmann I. P., Tinetti G., arXiv:1912.07759

Allard F., Hauschildt P. H., Alexander D. R., Tamanai A., Schweitzer A., 2001, ApJ, 556, 357

Barbato D., et al., 2020, A\&A, 641, A68

Barnes R., 2011, in Gargaud M., Amils R., Quintanilla J. C., Cleaves H. J., Irvine W. M., Pinti D. L., Viso M., eds., Encyclopedia of Astrobiology (Springer Berlin Heidelberg), pp 1395

Barker A. J., Ogilvie G. I., 2010, MNRAS, 404, 1849

Barros S. C. C., Boué G., Gibson N. P., Pollacco D. L., Santerne A., Keenan F. P., Skillen I., Street R. A., 2013, MNRAS, 430, 3032

Bean J. L., et al., 2018, PASP, 130, 114402

Bell T. J., et al., 2021, MNRAS, 504, 3316

Birkby J. L., et al., 2014, MNRAS, 440, 1470

Blecic J., et al., 2014, ApJ, 781, 116

Bolmont E., Gallet F., Mathis S., Charbonnel C., Amard L., Alibert Y., A\&A, 604, A113

Bonomo A.S., et al., 2017, A\&A, 602, A107

Chernov S. V., 2017, AstL., 43, 186

Choi J., et al., 2016, ApJ, 823, 102

Ciceri S. et al., 2013, A\&A, 557, A30

Claret A., 2004, A\&A, 424, 919
Collier Cameron A., Jardine M., 2018, MNRAS, 476, 2542

Collins K. A., Kielkopf J. F., Stassun K. G., Hessman F. V., 2017, AJ, 153, 77

Dosopoulou F., Naoz S., Kalogera V., 2017, ApJ, 844, 12

Dotter A., 2016, ApJS, 222, 8

Eastman J. D., et al., 2019, arXiv:1907.09480

Essick R., Weinberg N. N., 2016, ApJ, 816, 18

Ferraz-Mello S., Rodríguez A., Hussmann H., 2008, CeMDA, 101, 171

Fortney J. J., Lodders K., Marley M. S., Freedman R. S., 2008, ApJ, 678, 1419

Fortney J. J., Shabram M., Showman A. P., Lian Y., Freedman R. S., Marley M. S., Lewis N. K., 2010, ApJ, 709, 1396

Gallet F., Bolmont E., Mathis S., Charbonnel C., Amard L., 2017, A\&A, 604, A112

Gibbs A., et al., 2020, AJ, 159, 169

Gibson, N. P., Pollacco, D., Simpson, E. K., et al. 2008, A\&A, 492, 603

Gillon M., et al., 2006, A\&A, 459, 249

Goldreich P., Soter S., 1966, Icarus, 5, 375

Guillot T., 2010, A\&A, 520, A27

Hamer J. H., Schlaufman K. C., 2019, AJ, 158, 190

Hebb L., et al., 2009, ApJ, 693, 1920

Hoyer S., López-Morales M., Rojo P., Minniti D., Adams E. R., 2016, MNRAS, 455, 1334

Hut P., 1980, A\&A, 92, 167

Jackson B., Greenberg R., Barnes R., 2008, ApJ, 678, 1396

Jiang I.-G., Lai C.-Y., Savushkin A., Mkrtichian D., Antonyuk K., Griv E., Hsieh H.-F., Yeh L.-C., 2016, AJ, 151, 17

Kurucz R., 1979, ApJS, 40, 1

Kurucz R., 1993, ATLAS9 Stellar Atmosphere Programs and 2 km/s Grid. Kurucz CD-ROM No. 13

Lai D., 2012, MNRAS, 423,486

Levrard B., Winisdoerffer C., Chabrier G., 2009, ApJ, 692, L9

Lightkurve Collaboration, Cardoso J. V. d. M., et al., 2018, Lightkurve: Kepler and TESS time series analysis in Python, Astrophysics Source Code Library. http: //ascl .net/1812.013

Love A., 1944, A Treatise on the Mathematical Theory of Elasticity. Dover Books on Engineering Series (Dover Publications)

Luger R., Agol E., Foreman-Mackey D., Fleming, D. P., Lustig-Yaeger J., Deitrick R., 2019, AJ, 157, 64

Maciejewski G., et al., 2013, A\&A, 551, A108

Maciejewski G., et al., 2016, A\&A, 588, L6

Maciejewski G., et al., 2018a, AcA, 68, 371

Maciejewski G., Knutson H. A., Howard A. W., Isaacson H., FernándezLajús E., DiSisto R. P., Migaszewski C., et al., 2018b, AcA, 70, 1

Madhusudhan N., 2012, ApJ, 758, 36

Mancini L., et al., 2013a, A\&A, 551, A11

Mancini L., et al., 2013b, MNRAS, 430, 2932

Mancini L., et al., 2014, A\&A, 562, A126

Mancini L., et al., 2017, MNRAS, 465, 843

Matsumura S., Takeda G., Rasio F. A., 2008, ApJ, 686, L29

Matsumura S., Peale S. J., Rasio F. A., 2010, ApJ, 725, 1995

Mayor M., Queloz D., 1995, Nature, 378, 355

Murgas F., Pallé E., Zapatero Osorio M. R., Nortmann L., Hoyer S., CabreraLavers A., 2014, A\&A, 563, A41

Nikolov, N., Henning, Th., Koppenhoefer, J., et al. 2012, A\&A, 539, 159

Oberst T. E., et al., 2017, AJ, 153, 97

Ogilvie G. I., Lin D. N. C., 2007, ApJ, 661, 1180

Ogilvie G. I., 2014, ARA\&A, 52, 171

Paczynski B., 1971, ARA\&A, 9, 183

Pablo H., et al., 2017, MNRAS, 467, 2494

Patra K. C., Winn J. N., Holman M. J., Yu L., Deming D., Dai F., 2017, 154, 4

Patra K. C., et al., 2020, AJ, 159, 150

Penev K., Sasselov D. D., 2011, ApJ, 731, 67

Penev K., Jackson B., Spada F., Thom N., 2012, ApJ, 751, 96

Pont F., Husnoo N., Mazeh T., Fabrycky D., 2011, MNRAS, 414, 1278

Rasio F. A., Ford E. B., 1996, Science, 274, 954

Rasio F. A., Tout C. A., Lubow S. H., Livio M., 1996, ApJ, 470, 1187 
Ricci D., et al., 2017, PASP, 129, 064401

Ricci D., et al., 2020, arXiv:2011.13262

Ricker G. R., et al., 2015, J. Astron. Telesc. Instrum. Syst., 1, 014003

Sasselov D. D., 2003, ApJ, 596, 1327

Sing D. 2018, Astrophysics of Exoplanetary Atmosphere, eds., V. Bozza, L. Mancini, \& A. Sozzetti (Switzerland: Springer International Publishing), 3

Southworth, J., 2011, MNRAS, 417, 2166

Southworth J., 2012, MNRAS, 426, 1291

Southworth J., 2013, A\&A, 557, A119

Southworth J., 2008, MNRAS, 386, 1644

Southworth J., et al., 2009, MNRAS, 396, 1023

Southworth J., et al., 2010, MNRAS, 408, 1680

Southworth J., Bruni I., Mancini, L., Gregorio J., 2012a, MNRAS, 420, 2580

Southworth J., Mancini L., Maxted P. F. L., Bruni I., Tregloan-Reed J., Barbieri M., Ruocco N., Wheatley P. J., 2012b, MNRAS, 422, 3099

Southworth J., et al., 2016, MNRAS, 457, 4205

Southworth J., et al., 2019, MNRAS, 490, 4230

Tregloan-Reed J., Southworth J., Tappert C., 2013, MNRAS, 428, 3671

Tregloan-Reed J., et al., 2015, MNRAS, 450, 1760

Tregloan-Reed J., Unda-Sanzana E., 2019, A\&A, 630, A114

Tregloan-Reed J., Unda-Sanzana E., 2021, arXiv:2103.16539

Turner J. D., Ridden-Harper A., Jayawardhana R., 2021, AJ, 161, 72

Wilkins A. N., Delrez L., Barker A. J., Deming D., Hamilton D., Gillon M., Jehin E., 2017, ApJ, 836, L24

Winn, J. N., Holman, M. J., Torres, G., et al. 2008, ApJ, 683, 1076

Yee S. W., et al., 2020, ApJ, 888, L5

\section{APPENDIX A: SIMULTANEOUS LIGHT CURVES \\ APPENDIX B: ZOOM IN TO THE O-C PLOT APPENDIX C: FIT OF THE PHASE CURVE}

This paper has been typeset from a $\mathrm{T}_{\mathrm{E}} \mathrm{X} / \mathrm{LAT} \mathrm{EX}$ file prepared by the author.
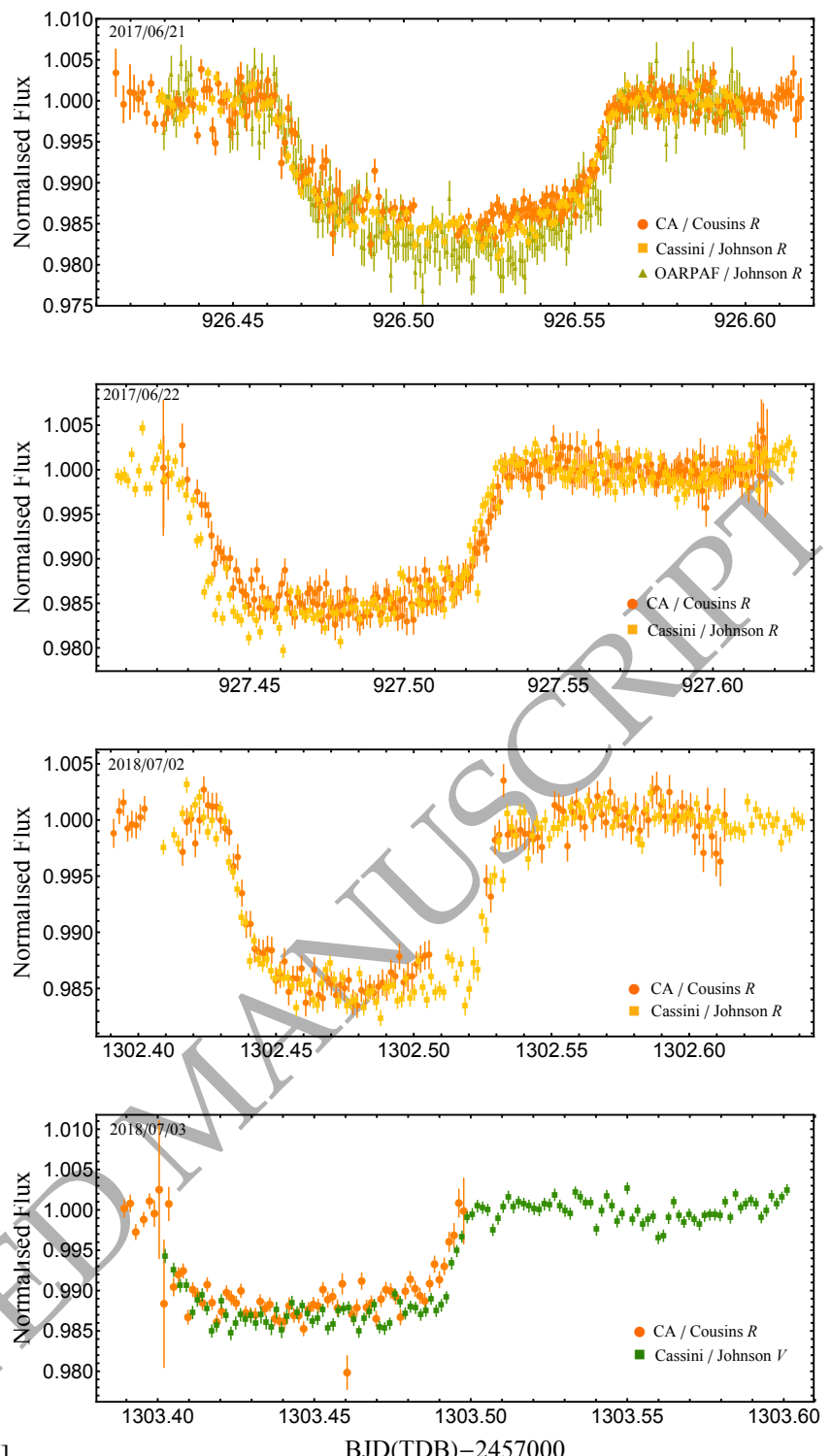

[H]

Figure A1. The fours transit events that were simultaneously observed with three telescopes (top panel) and two telescopes bottom panels. 

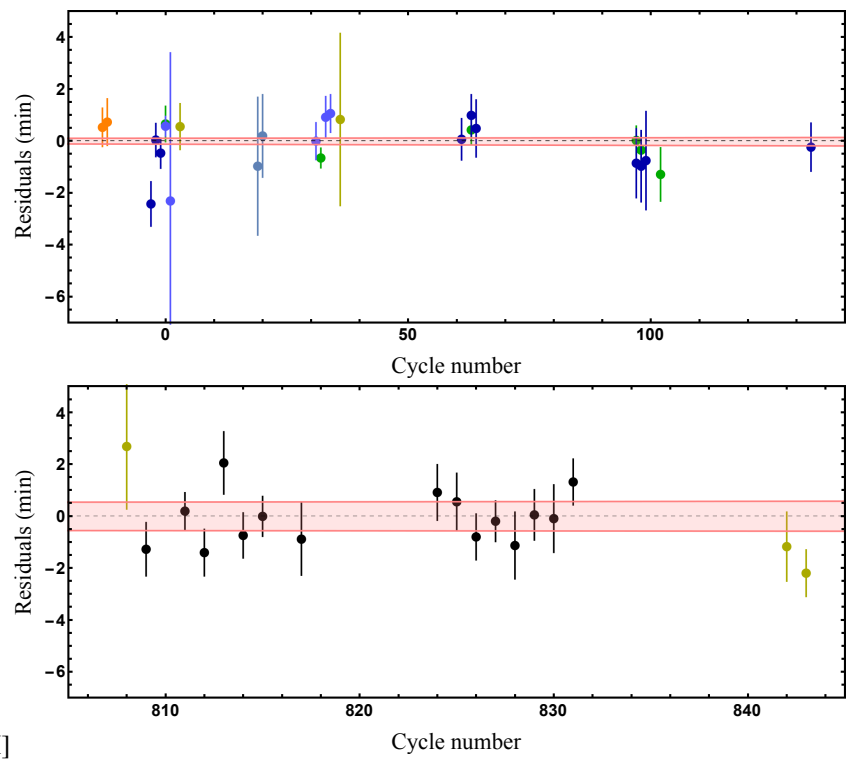

$[\mathrm{H}]$

Figure B1. Zooms in to the best sampled region (top panel) and in that covered by TESS (bottom panel). The points are coloured as in Fig 10.

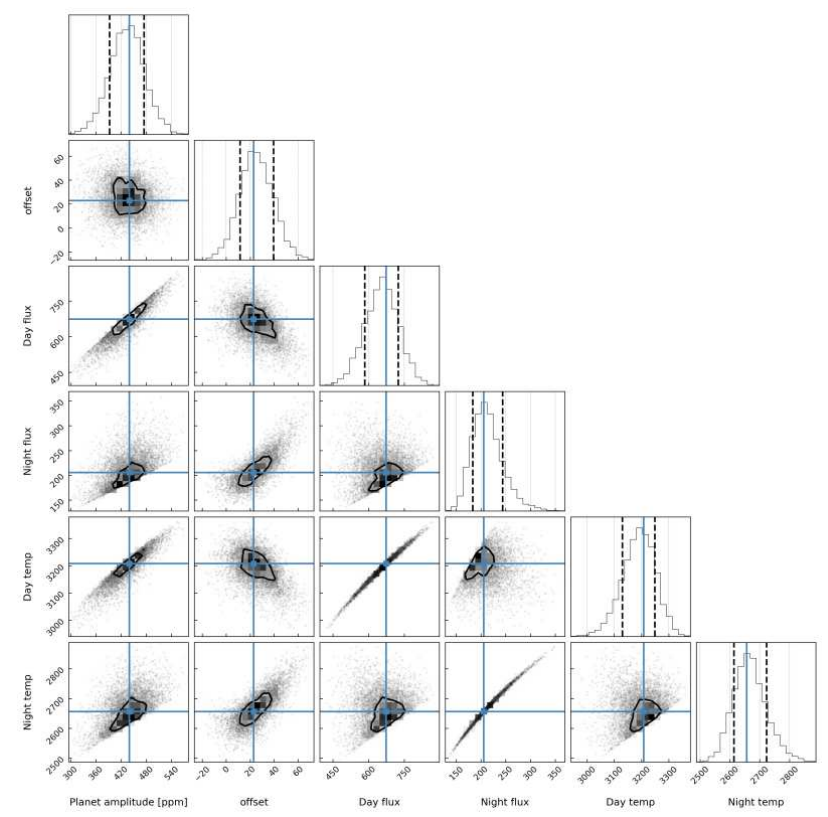

Figure C1. Distributions of the posterior values over the model parameters from a Starry computation with 8000 draw iterations. Mean values are highlighted with blue lines, while the contour levels of the joint probability densities have been drawn at $1 \sigma$ distance. 